\title{
A manually annotated Actinidia chinensis var. chinensis (kiwifruit) genome highlights the challenges associated with draft genomes and gene prediction in plants
}

Sarah M. Pilkington ${ }^{1}$, Ross Crowhurst ${ }^{1}$, Elena Hilario ${ }^{1}$, Simona Nardozza', Lena Fraser ${ }^{1}$, Yongyan Peng ${ }^{1,2}$, Kularajathevan Gunaseelan', Robert Simpson ${ }^{3}$, Jibran Tahir ${ }^{3}$, Simon C. Deroles ${ }^{3}$, Kerry Templeton ${ }^{1}$, Zhiwei Luo ${ }^{1}$, Marcus Davy ${ }^{4}$, Canhong Cheng ${ }^{1}$, Mark McNeilage ${ }^{1}$, Davide Scaglione ${ }^{5}$, Yifei Liu', Qiong Zhang ${ }^{7}$, Paul Datson', Nihal De Silva ${ }^{1 \wedge}$, Susan E. Gardiner ${ }^{3}$, Heather Bassett ${ }^{3}$, David Chagné $^{3}$, John McCallum ${ }^{8}$, Helge Dzierzon ${ }^{3}$,

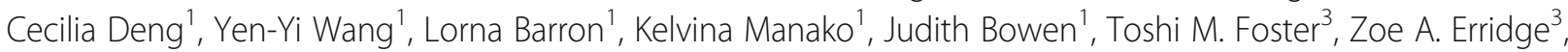

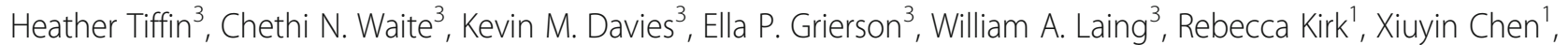
Marion Wood ${ }^{1}$, Mirco Montefiori ${ }^{1}$, David A. Brummell ${ }^{3}$, Kathy E. Schwinn ${ }^{3}$, Andrew Catanach ${ }^{8}$, Christina Fullerton ${ }^{1}$, Dawei Li', Sathiyamoorthy Meiyalaghan ${ }^{8}$, Niels Nieuwenhuizen ${ }^{1}$, Nicola Read ${ }^{2}$, Roneel Prakash', Don Hunter ${ }^{3}$, Huaibi Zhang ${ }^{3}$, Marian McKenzie 3 , Mareike Knäbel ${ }^{3}$, Alastair Harris ${ }^{2}$, Andrew C. Allan ${ }^{1,2}$, Andrew Gleave ${ }^{1}$, Angela Chen'2, Bart J. Janssen ${ }^{1}$, Blue Plunkett', Charles Ampomah-Dwamena', Charlotte Voogd', Davin Leif ${ }^{1,2}$, Declan Lafferty ${ }^{2}$, Edwige J. F. Souleyre ${ }^{1}$, Erika Varkonyi-Gasic ${ }^{1}$, Francesco Gambi ${ }^{1}$, Jenny Hanley ${ }^{2}$, Jia-Long Yao ${ }^{1}$, Joey Cheung ${ }^{2}$, Karine M. David², Ben Warren', Ken Marsh', Kimberley C. Snowden', Kui Lin-Wang1', Lara Brian', Marcela Martinez-Sanchez', Mindy Wang ${ }^{1}$, Nadeesha lleperuma', Nikolai Macnee', Robert Campin', Peter McAtee', Revel S. M. Drummond', Richard V. Espley', Hilary S. Ireland', Rongmei Wu', Ross G. Atkinson', Sakuntala Karunairetnam', Sean Bulley ${ }^{4}$, Shayhan Chunkath², Zac Hanley' ${ }^{1}$ Roy Storey ${ }^{4}$, Amali H. Thrimawithana', Susan Thomson ${ }^{8}$, Charles David ${ }^{8}$, Raffaele Testolin ${ }^{5,9}$, Hongwen Huang ${ }^{6,7}$, Roger P. Hellens ${ }^{10}$ and Robert J. Schaffer ${ }^{1,2^{*}}$

\section{Abstract}

Background: Most published genome sequences are drafts, and most are dominated by computational gene prediction. Draft genomes typically incorporate considerable sequence data that are not assigned to chromosomes, and predicted genes without quality confidence measures. The current Actinidia chinensis (kiwifruit) 'Hongyang' draft genome has $164 \mathrm{Mb}$ of sequences unassigned to pseudo-chromosomes, and omissions have been identified in the gene models. (Continued on next page)

\footnotetext{
* Correspondence: robert.schaffer@plantandfood.co.nz

'Deceased

'The New Zealand Institute for Plant \& Food Research Ltd (PFR), Private Bag

92169, Auckland 1142, New Zealand

${ }^{2}$ School of Biological Sciences, University of Auckland, Private Bag 92019,

Auckland 1142, New Zealand

Full list of author information is available at the end of the article
}

(C) The Author(s). 2018 Open Access This article is distributed under the terms of the Creative Commons Attribution 4.0 International License (http://creativecommons.org/licenses/by/4.0/), which permits unrestricted use, distribution, and reproduction in any medium, provided you give appropriate credit to the original author(s) and the source, provide a link to the Creative Commons license, and indicate if changes were made. The Creative Commons Public Domain Dedication waiver (http://creativecommons.org/publicdomain/zero/1.0/) applies to the data made available in this article, unless otherwise stated. 
(Continued from previous page)

Results: A second genome of an A. chinensis (genotype Red5) was fully sequenced. This new sequence resulted in a $554.0 \mathrm{Mb}$ assembly with all but $6 \mathrm{Mb}$ assigned to pseudo-chromosomes. Pseudo-chromosomal comparisons showed a considerable number of translocation events have occurred following a whole genome duplication (WGD) event some consistent with centromeric Robertsonian-like translocations. RNA sequencing data from 12 tissues and ab initio analysis informed a genome-wide manual annotation, using the WebApollo tool. In total, 33,044 gene loci represented by 33,123 isoforms were identified, named and tagged for quality of evidential support. Of these 3114 (9.4\%) were identical to a protein within 'Hongyang' The Kiwifruit Information Resource (KIR V2). Some proportion of the differences will be varietal polymorphisms. However, as most computationally predicted Red5 models required manual re-annotation this proportion is expected to be small. The quality of the new gene models was tested by fully sequencing 550 cloned 'Hort 16A' cDNAs and comparing with the predicted protein models for Red5 and both the original 'Hongyang' assembly and the revised annotation from KIR v2. Only $48.9 \%$ and $63.5 \%$ of the cDNAs had a match with $90 \%$ identity or better to the original and revised 'Hongyang' annotation, respectively, compared with 90.9\% to the Red5 models.

Conclusions: Our study highlights the need to take a cautious approach to draft genomes and computationally predicted genes. Our use of the manual annotation tool WebApollo facilitated manual checking and correction of gene models enabling improvement of computational prediction. This utility was especially relevant for certain types of gene families such as the EXPANSIN like genes. Finally, this high quality gene set will supply the kiwifruit and general plant community with a new tool for genomics and other comparative analysis.

Keywords: Manual annotation, Genome sequencing, Actinidia chinensis

\section{Background}

The time, effort and cost of obtaining whole genome sequences has reduced dramatically since the publishing of the first whole plant genome for Arabidopsis thaliana in 2000 [1]. As a result, more than 100 plant genomes have now been sequenced, including those for a number of fruit crops of worldwide horticultural importance, such as Vitis vinifera (grape) [2], Carica papaya (papaya) [3], Malus x domestica (apple) [4], Fragaria vesca (strawberry) [5], Solanum lycopersicum L. (tomato) [6], Musa acuminata (banana) [7], Citrus sinensis (orange) [8], and Pyrus comunis L. (European pear) [9]. However, there are still many challenges for plant genome assembly including fragmentation, large numbers of contigs, misassembly and the polyploid nature of many plant species, contribute to large amounts of sequence remaining unassigned to chromosomes in many genomes and thus impact the quality of the gene annotation within them $[10,11]$. This is now being addressed with new improved versions of genomes appearing in the literature $[12,13]$.

The A. chinensis draft genome [14] represented a significant step forward for kiwifruit researchers. However, as is typical for draft whole genome sequences, a significant proportion of the scaffolds was unassigned to chromosomes, and mis-assemblies have been subsequently identified: Scaglione and colleagues were the first to identify scaffold misplacements and revealed significant discrepancies that indicated scaffold mis-assignments in chromosomes (Chr) $6,10,16,18,19,20$ and 21 [15]. The most significant discrepancy was $4.5 \mathrm{Mb}$ of scaffolds attributed to Chr10 that mapped unambiguously to Chr16. Zhang and colleagues also reported the possibility of scaffold anchoring errors and suggested that the draft genome contained many interchromosomal misplacements [16].

The 'Hongyang' genome sequence was annotated using a combination of computer annotation, Expressed Sequence Tag (EST) sequence information from publicly available databases and in-house RNA sequencing (RNA-Seq), which resulted in 39,040 predicted genes [14]. The predicted 'Hongyang' gene models have recently been shown to omit key published EXPANSIN (EXP) genes [17]. These missing genes may be due to errors in the genome assembly itself, caused by introduced stop codons nullifying a prediction. Although, the majority (97\% [14]) of the EXP ESTs are found in the 'Hongyang' genome sequence, most were not found in the annotated gene list. This indicates that the rules set for inclusion of a predicted gene in the published gene set may have been too conservative, and that re-annotation of the genome sequence is necessary to improve representation within the predicted gene set currently available. To that end, an extensive revision of the 'Hongyang' annotation was performed by Yue and colleagues [18]. Their efforts yielded a much improved annotation for 'Hongyang' as well as providing further information on splice variants, predicted metabolic pathways and protein-protein interactions. In other species, gene models constantly evolve, with Arabidopsis now on its 11th release [19]. Computational re-annotation [20] of the strawberry genome [5] increased the total number of gene model predictions by 2286 predictions.

Most commonly, genome annotation methods have been computationally derived $[4,5]$, although research communities are increasingly combining computer annotation methods with manual annotation that allows researchers to improve individual gene models within the genome. The 
software package WebApollo [21] has been used for gene annotation initiatives in a number of species, including Caenorhabditis elegans [22], yeast [23] and honey bee [21], as well as Arabidopsis [24]. Community annotation leverages the expert knowledge within a community to identify and correct errors in computational predictions and insert models missed by those computational approaches. At present, manual curation of genomes is rare, but could become increasingly common as researchers recognise that computational assembly and annotation alone are not sufficient.

In our study, three research teams pooled genetic mapping and sequence resources to generate a genome of a second $A$. chinensis genotype, Red5, with higher homozygosity than 'Hongyang'. This information was combined with EST sequencing results [25] and RNA-Seq data and made available to annotators via WebApollo to facilitate manual annotation of the new genome. The whole genome was manually annotated, resulting in what we believe to be a considerable improvement in allocating previously unallocated regions and in gene model quality as compared to existing resources.

\section{Results}

Assembly of a second genome of Actinidia chinensis var. chinensis

To generate a new Actinidia chinensis genome, a diploid F3 sibcross individual Red5, with a predicted inbreeding coefficient of $37.5 \%$, was chosen for sequencing (Fig. 1). An anytag-based assembly of paired-end Illumina reads generated 46,117,212 fragments with an N50 of 275 bases [26]. Assembly of these fragments using a long insert library (Roche 454 GS-FLX - $4 \mathrm{~kb}$ ) using Newbler produced 39,868 contigs. Subsequent stepwise scaffolding using SSPACE2 [27] with Illumina long-range insert libraries of 4, 9 and $13 \mathrm{~kb}$ yielded 39,825, 8688 and 3887 scaffolds, respectively. After two iterations of gap closure the final assembly consisted of 3887 scaffolds with a total length of $550.5 \mathrm{Mb}$. The N50 was $623.8 \mathrm{~kb}$ with L50 of 240 scaffolds, an N90 of $140.7 \mathrm{~kb}$ with L90 of 941 scaffolds, and $3.57 \% \mathrm{~N}$ content, with the longest scaffold being $4.43 \mathrm{Mb}$.

The genetic linkage map of Scaglione and colleagues [15], which included markers from Fraser and colleagues [28], augmented by BLAST walking comparison between Red5 and scaffolds of 'Hongyang' enabled anchoring of 2727 scaffolds (Table 1) comprising $547.9 \mathrm{Mb}$, to 29 linkage groups. The remaining 1206 unanchored scaffolds containing $5.91 \mathrm{Mb}$ with an N50 of $5.36 \mathrm{~kb}$ were concatenated to form a composite entity hereafter referred to as 'Chr30' for the purposes of subsequent manual annotation of the entire genome sequence. Note, upon submission to NCBI Genbank scaffolds assigned to 'Chr30' were submitted as individual (non-concatenated) scaffolds according to NCBI

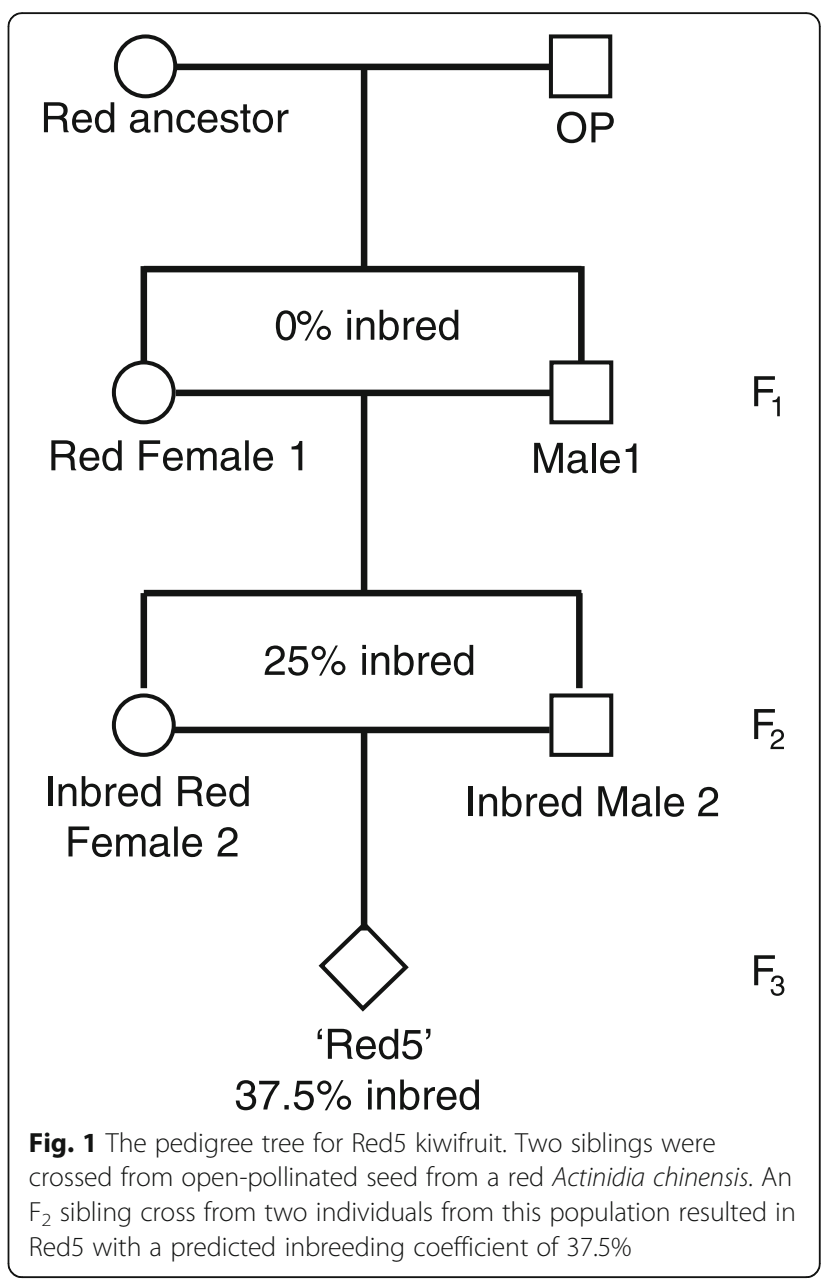

Genbank submission policy. Estimates of genome size based on K-mer analysis indicated a genome size of $705 \mathrm{Mb}$ (preQC) or $742 \mathrm{Mb}$ (jellyfish). These align with estimates from flow cytometry [29] that report the genome to be $758 \mathrm{Mb}$ in size. The assembly therefore represents $\sim 73 \%$ of the estimated genome size with $98.9 \%$ of assembled scaffolds (72\% of the estimated genome size) assigned to chromosomes. This is a considerable improvement from the original 'Hongyang' draft genome, which had $164 \mathrm{Mb}$ unassigned (Fig. 2).

\section{Evaluation of genome assembly}

To evaluate genome assembly accuracy we assessed mapping back of paired end reads to the assembly and compared the assembled contigs for 22 clones from a BAC library of $A$. chinensis Red Female 1 (Fig. 1). These contigs resulted from sequencing using a different technology (454) and different assembly path (Newbler). Rates of discordant alignment of input paired end reads mapping once to the whole genome sequence was low (0.33 to 2.24\%) (Additional file 1). Alignment of these BAC clone contig assemblies to chromosome 25 supported the assembly of 
Table 1 Construction metrics for the Actinidia chinensis genome

\begin{tabular}{|c|c|c|c|c|c|c|c|}
\hline \multirow[t]{2}{*}{ Chromosome } & \multirow{2}{*}{$\begin{array}{l}\text { Size } \\
(\mathrm{Mb})\end{array}$} & \multirow{2}{*}{$\begin{array}{l}\text { Number of } \\
\text { Scaffolds }\end{array}$} & \multirow{2}{*}{$\begin{array}{l}\text { Scaffold } \\
\text { N50 (Kb) }\end{array}$} & \multirow{2}{*}{$\begin{array}{l}\text { Longest } \\
\text { Scaffold (Mb) }\end{array}$} & \multicolumn{3}{|c|}{ Manually Annotated Genes } \\
\hline & & & & & $\begin{array}{l}\text { Number of } \\
\text { Genes }\end{array}$ & $\begin{array}{l}\text { Number of } \\
\text { Transcripts }\end{array}$ & $\begin{array}{l}\text { Genes } \\
\text { Per } 100 \mathrm{~Kb} \\
\end{array}$ \\
\hline 1 & 18.6 & 94 & 595.4 & 1.51 & 1125 & 1133 & 6.06 \\
\hline 2 & 14.6 & 83 & 536.6 & 1.41 & 1091 & 1094 & 7.46 \\
\hline 3 & 21.7 & 92 & 1154.9 & 2.96 & 1726 & 1729 & 7.94 \\
\hline 4 & 13.8 & 75 & 420.4 & 2.39 & 781 & 781 & 5.67 \\
\hline 5 & 18.6 & 113 & 655.2 & 4.44 & 960 & 976 & 5.16 \\
\hline 6 & 17.4 & 109 & 474.8 & 1.56 & 1059 & 1069 & 6.09 \\
\hline 7 & 20.0 & 99 & 666.0 & 1.82 & 1068 & 1075 & 5.33 \\
\hline 8 & 26.1 & 134 & 779.6 & 1.68 & 1448 & 1459 & 5.55 \\
\hline 9 & 16.6 & 66 & 598.4 & 1.95 & 1118 & 1123 & 6.74 \\
\hline 10 & 19.3 & 111 & 413.0 & 0.95 & 986 & 994 & 5.10 \\
\hline 11 & 16.9 & 74 & 520.3 & 1.26 & 1119 & 1137 & 6.63 \\
\hline 12 & 19.2 & 122 & 480.2 & 1.42 & 1053 & 1054 & 5.49 \\
\hline 13 & 19.5 & 77 & 926.8 & 1.65 & 1388 & 1402 & 7.11 \\
\hline 14 & 17.9 & 75 & 651.2 & 2.32 & 1106 & 1129 & 6.18 \\
\hline 15 & 15.9 & 67 & 707.9 & 2.13 & 1106 & 1121 & 6.94 \\
\hline 16 & 23.8 & 152 & 388.5 & 1.46 & 1252 & 1255 & 5.26 \\
\hline 17 & 17.4 & 100 & 387.2 & 0.91 & 931 & 934 & 5.34 \\
\hline 18 & 20.7 & 85 & 850.4 & 2.80 & 1213 & 1219 & 5.85 \\
\hline 19 & 15.4 & 117 & 345.9 & 2.12 & 653 & 656 & 4.24 \\
\hline 20 & 17.9 & 89 & 512.5 & 1.53 & 1055 & 1056 & 5.88 \\
\hline 21 & 17.3 & 71 & 871.2 & 2.16 & 1046 & 1064 & 6.04 \\
\hline 22 & 18.9 & 105 & 462.5 & 1.42 & 1092 & 1096 & 5.76 \\
\hline 23 & 27.7 & 79 & 802.6 & 2.15 & 2324 & 2327 & 8.39 \\
\hline 24 & 17.8 & 59 & 1032.0 & 3.02 & 1198 & 1201 & 6.72 \\
\hline 25 & 19.6 & 83 & 966.1 & 2.45 & 1008 & 1010 & 5.14 \\
\hline 26 & 20.4 & 79 & 1086.4 & 3.41 & 1237 & 1247 & 6.07 \\
\hline 27 & 21.0 & 112 & 461.8 & 1.27 & 1013 & 1040 & 4.83 \\
\hline 28 & 15.8 & 75 & 964.5 & 2.80 & 1011 & 1012 & 6.38 \\
\hline 29 & 18.0 & 130 & 402.3 & 0.69 & 993 & 1002 & 5.53 \\
\hline Total for Chrs 1-29 & 548.0 & 2727 & 575.6 & 4.44 & 33,160 & 33,395 & 6.05 \\
\hline Unassigned 30 & 5.9 & 1206 & 5.4 & 0.66 & 97 & 97 & 1.64 \\
\hline Total & 553.9 & 3933 & & & 33,257 & 33,492 & 6.00 \\
\hline
\end{tabular}

Red5. The alignments (Additional file 2) show a close correspondence between the $\mathrm{BAC}$ clone assemblies and the whole genome assembly of Red5 in this region of chromosome 25. Additionally, the alignment of read pairs from the 9Kb LIMP library was assessed and visualised using hagfish_blockplot from the 'hagfish' software (https://github. $\mathrm{com} / \mathrm{mfiers} /$ hagfish/). The majority of alignments displayed green indicating the read pairs from the $9 \mathrm{~Kb}$ LIMP library aligned to the chromosome sequences with the default bounds determined by 'hagfish' (Additional file 3). As expected for assembly from short read data there were also regions depicted in pinkish-red suggesting that the mate pairs aligned to the genome in these regions outside the expected distances. Such regions will occur for example when repeats are compressed into a consensus leading to a compression in the whole genome assembly sequence relative to the physical genome sequences.

To evaluate genome completeness BUSCO analysis [30] was undertaken. For purposes of comparison, these same analyses were repeated using the published chromosomal sequences for 'Hongyang' [14]. Red5 contained 1364 (94.7\%) 'complete' BUSCOs, of which 1022 (75.0\%) were reported as 

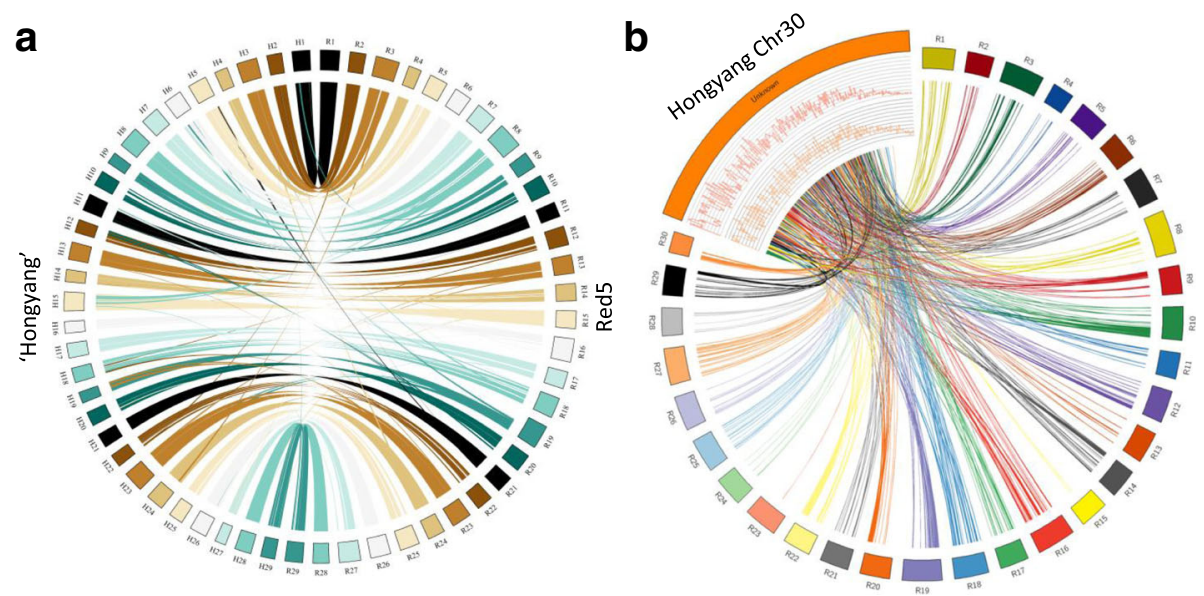

Fig. 2 a. Genome construction with respect to the original submitted Actinidia chinensis 'Hongyang' genome, showing the rearrangement of some assigned chromosomes and $\mathbf{b}$. the distribution of unallocated sequence (Chromosome 30 ) to the rest of the genome

'complete and single-copy', while $342(25 \%)$ were reported as 'complete and duplicated' with 27 reported as fragmented and 49 reported as missing. In comparison, 'Hongyang' contained 1358 (94.3\%) 'complete' BUSCOs, with $22(1.5 \%)$ reported as fragmented and $60(4.2 \%)$ reported as missing. Of the BUSCOs reported as 'complete' in 'Hongyang', 987 (68. $5 \%)$ were reported as 'complete and single copy', while 371 were reported as 'complete and duplicated'. When the 47,384 A. chinensis EST sequences in NCBI GenBank were mapped to the Red5 chromosomes, only 580 had no homology, with 2368 ESTs aligned at less than $74 \%$ match. In comparison, when the ESTs were aligned to the published chromosomes of 'Hongyang', 3295 had no homology to any region, suggesting Red5 and Hongyang have a comparable gene space assembly. When RNA-Seq data from a range of tissues (Table 2) were mapped to the chromosome assembly of Red5, an average of $91.95 \%$ of 316.2 million RNA-Seq reads mapped uniquely (ranging from $88.19 \%$ to $94.56 \%$ for

Table 2 Tissue for RNA-Seq data for the Actinidia chinensis genome

\begin{tabular}{|c|c|}
\hline Red5 tissue description & Number of reads \\
\hline tissue culture whole plant & $16 \mathrm{M}$ \\
\hline orchard plant growing bud & $16 \mathrm{M}$ \\
\hline orchard plant flower & $19 \mathrm{M}$ \\
\hline orchard fruit 0 DAFB ${ }^{1}$ & $18 \mathrm{M}$ \\
\hline orchard fruit $14 \mathrm{DAFB}^{1}$ & $0.2 \mathrm{M}$ \\
\hline orchard fruit $60 \mathrm{DAFB}^{1}$ & $14 \mathrm{M}$ \\
\hline orchard fruit $76 \mathrm{DAFB}^{1}$ & $59 \mathrm{M}$ \\
\hline orchard fruit 139 DAFB $^{1}$ & $60 \mathrm{M}$ \\
\hline glasshouse pot plant root tip & $56 \mathrm{M}$ \\
\hline glasshouse pot plant root main & $58 \mathrm{M}$ \\
\hline
\end{tabular}

${ }^{1} D A F B$ days after full bloom the different tissues). $6.42 \%$ total reads mapped to multiple locations.

Global analysis comparing the new genome with itself revealed areas of similarity among different regions of the chromosomes (Fig. 3a). When these duplicated chromosomes were examined more closely it was found that many appeared to have Robertsonian-like centromeric translocations. This could be clearly seen, for example, in the duplicated Chr1, which showed homology to half of Chr8 and half of Chr9 (Fig. 3b). The paired chromosomes were arranged sequentially (Fig. 3c) and all but two had at least one translocation event. The only nontranslocated chromosomes were Chr4 (homeologous to Chr21) and Chr2 (homeologous to Chr3) (Fig. 3c).

\section{Manual curation of the predicted gene models}

To develop gene models for the new genome, a WebApollo tool [21] was populated with the new genome sequence and the following tracks of evidence were added:

1) A scaffold assembly quality track which identified single $\mathrm{N}$ insertions, indicative of construction anomalies, and $\mathrm{N}$ repeats that marked the edges of scaffold boundaries

2) A repeat masker track that identified repetitive elements, including transposons

3) Computational gene prediction tracks consisting of the original 'Hongyang' gene models, and an ab initio gene prediction scan of the Red5 genome

4) ESTs from published EST sequencing libraries, and 12 tracks of RNA-Seq from diverse tissues from Red5 (Table 2).

An international consortium of annotators synthesised this information to produce a new gene model for each gene along the genome. 
a

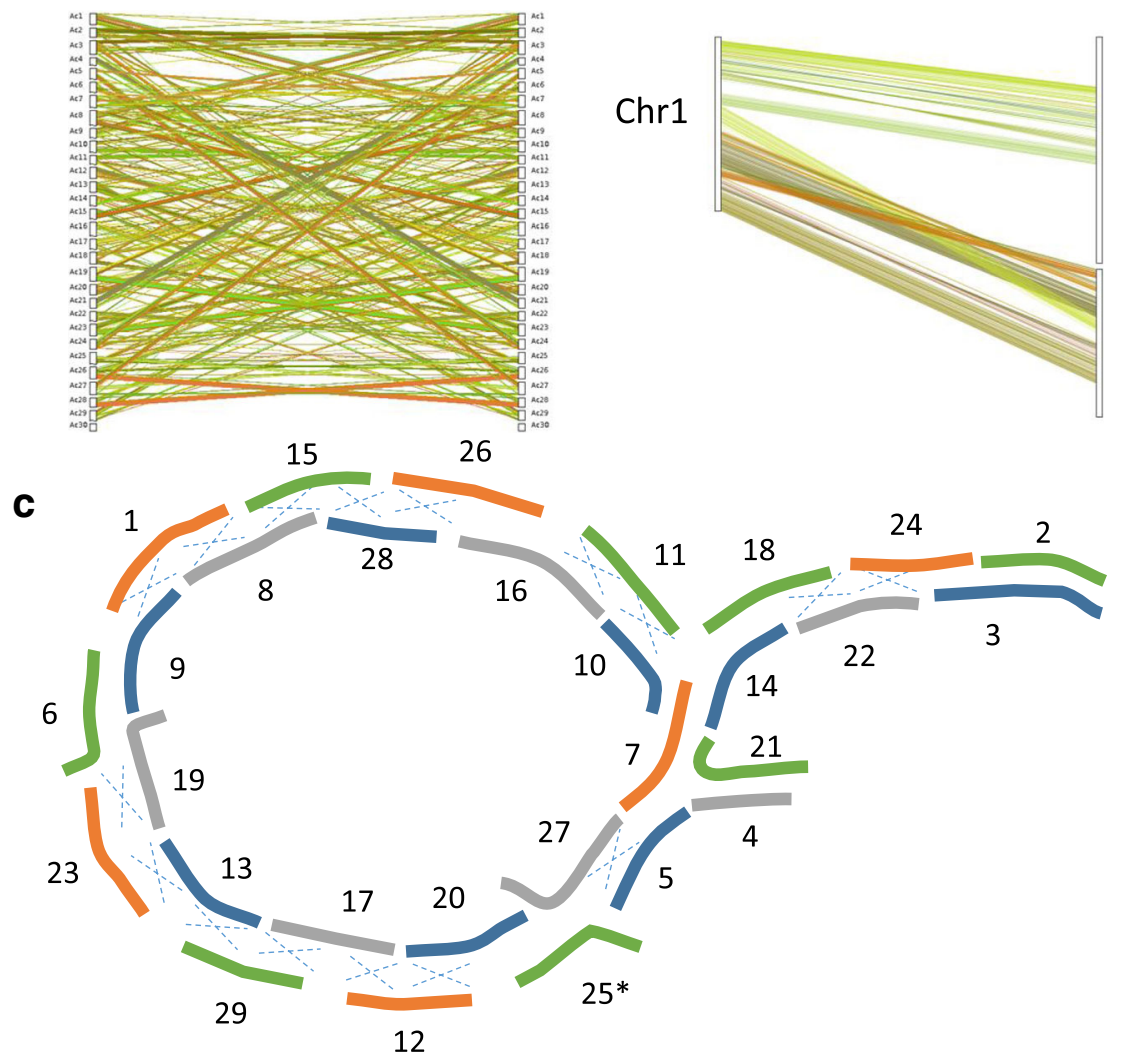

b

Chr8

Chr9

Fig. 3 Duplicated regions in Actinidia chinensis Red5. a. whole genome lined up with whole genome at 85\% homology. b. Alignments of Chr9 and Ch8 with Chr1, suggesting a translocation event has occurred. c. A schematic of the translocated chromosomes. A dashed " $x$ " suggests a Robertsonian translocation has occurred at the centromeric regions. Chromosomes not aligned with a dashed " $x$ " show regions of homology i.e. other proposed chromosomal rearrangements. Asterisk marks the sex chromosome (Chr25)

During the annotation process, some models were relatively simple to predict while others were more difficult. Easy-to-annotate models had strong RNA-Seq support, clear intron-exon structures and a gradation in the number of RNA-Seq reads at the beginning and the end of the gene. These genes also often had an accurate computationally predicted gene model and had a good BLASTP match from GenBank that covered the majority of the gene. Conversely, there were many regions of the genome that were very difficult to annotate. These harder-to-annotate regions had conflicting, patchy, or even no RNA-Seq evidence, combined with conflicting or absent computational gene models (Fig. 4). The complexity of these gene regions was often confounded by genome structure caused by repetitive elements and/or anomalies in the genome sequence construction, as observed by a single "N" or scaffold boundaries in the quality track. Within the genome, the whole spectrum of combinations of these challenges were identified with occasional loss of open reading frame caused by the anomalies. To address this variation in confidence for each model, a quality tag was added to each of the gene models. A strongly supported high quality gene model was given a Q2 tag, while a model that was suspected to be incomplete or contain other errors was given a Q1 tag. Further tags based on whether the BLASTP alignment suggested it was full length $(\mathrm{F})$ or a partial $(\mathrm{P})$ gene were also added. A Q0 tag was given to computationally predicted genes that exhibited peptide homology to a GenBank protein, but no RNA-Seq evidence. Following the first pass of manual annotation, it was found that there was a high degree of variability among the 93 annotators in their interpretation of the evidence to create the models, especially for loci where evidence was ambiguous, sparse, or apparently contradictory. To address this variation, a second pass of the genome was undertaken by a smaller group of 'expert' annotators to standardise the annotation.

The manual annotation identified 33,044 gene loci, with 33,123 protein isoforms. The mean coding length of annotated genes was 1278 bases, while the mean locus length was 6071 bases (range 282-92,048 bases). Of the 33,123 loci, 32,967 (99.5\%) were manually annotated with a single isoform, while 76 were annotated with two 
a

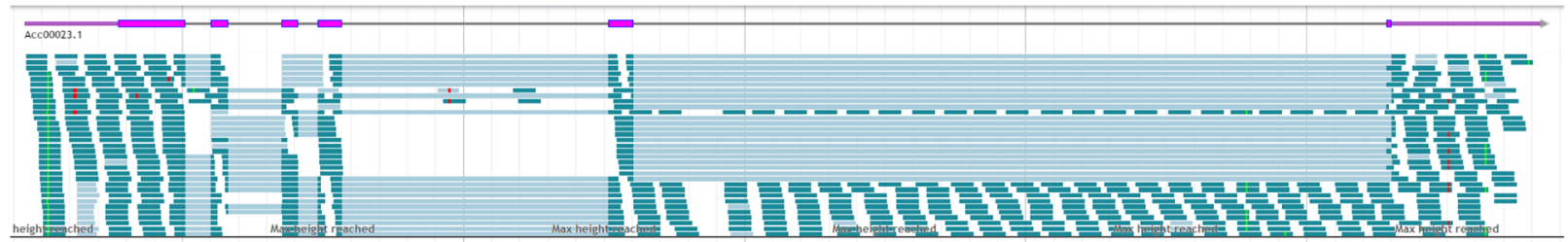

b

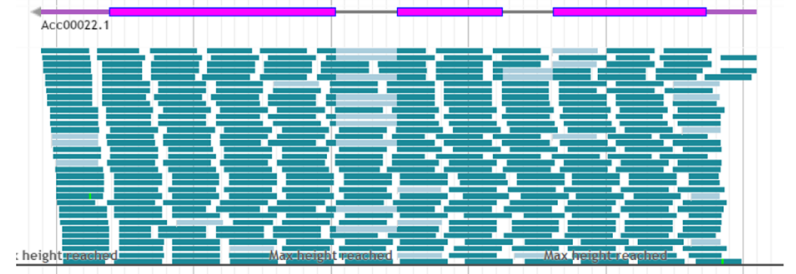

C

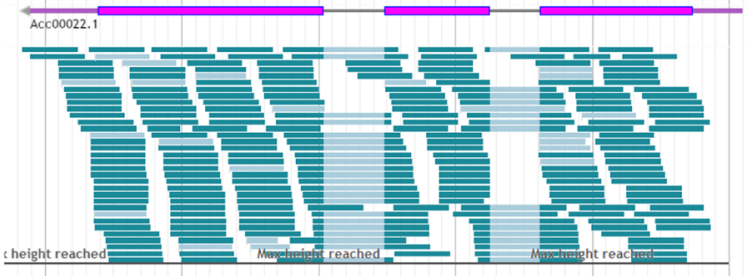

d

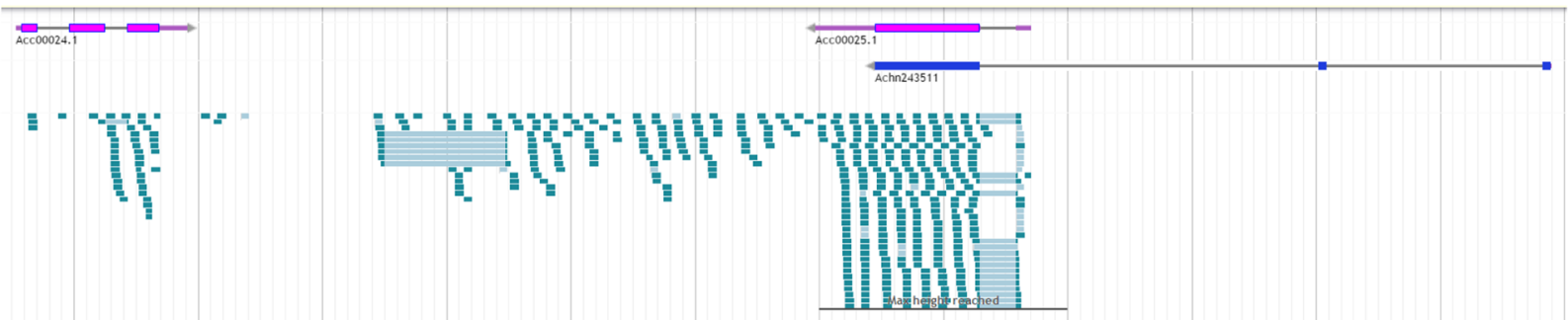

Fig. 4 Examples of genes with transcription, and no transcription in intronic regions highlighting some of the challenges associated with manual annotation. a RNA-Seq reads in a single intron of gene Acc00023. $\mathbf{b}$ and $\mathbf{c}$ a comparison of RNA-Seq reads in two independent RNA-Seq libraries of gene Acc00022. B) RNA-Seq from a root library showing transcription across the intron and C) RNA-Seq from a flower library showing no intronic RNA-Seq reads. $\mathbf{d}$ Transcription between gene models Acc00024 and Acc00025, which could not be constructed into a gene model; the Actinidia chinensis 'Hongyang' predicted model line-up Achn243511 is shown in blue

isoforms. One locus was annotated with four isoforms respectively. It should be noted that for loci with potentially more than one isoform individual isoforms were only annotated where they were cleanly defined from the RNA-Seq evidence. Otherwise a single model was submitted by annotators. Therefore it is likely that there will be further isoforms defined as further evidence is obtained. Of the 33,123 loci, 2485 were annotated by the community annotators as partials, with 1057 annotated as having $5^{\prime}$ truncations, and 817 as having $3^{\prime}$ truncations, while 611 loci were annotated as being partial without an indication of truncation direction. The total number of coding sequence regions (CDS) in annotated genes was 181,135 and contained $42.3 \mathrm{Mb}$ of genome sequence $(5.58 \%$ of the estimated genome size). There were 6514 loci $(19.7 \%)$ annotated as containing a single CDS. The mean length of CDS regions in single CDS loci was 1004 bases. In comparison, the mean CDS length for all loci was 233 bases. The mean intron length within the coding regions of multiple exon-containing loci was 886 bases. The minimum intron length was 22 bases. The corresponding quality scores were assessed and $83.16 \%$ of gene models had a Q2 score, $14.62 \%$ had a Q1 score and 2.18\% had a Q0 score.

Since genomes are always evolving, it is likely that different genomic structures will be observed across Actinidia species. Additionally, new improved versions of the genome annotation will be developed that incorporate the last $6 \mathrm{Mb}$ of unassigned fragments. To address this, we named our genes each with a unique name that will be enduring and independent of chromosome location. We have used the nomenclature AccXXXXX with splice variants appended as '.1' and '.2' etc., and then appended chromosome location as a descriptor which can subsequently be changed, without changing the unique name of the gene. To assist the kiwifruit research community we provide a conversion table for the best reciprocal matches between our gene set and that within the Kiwifruit Information Resource (KIR) [18] as Additional file 4 and will also make this data available via our Git Repository (https://github.com/PlantandFoodResearch/Red5_WGS_Manual_Annotation).

In addition to the location, the sequence length, sequence type (cds, cdna, peptide), and manual annotation 
quality score were appended along with an internal database identifier and functional description. The manual quality score also contained the F and P labels based on BLAST match, so a point to note, is a gene with a manual quality score of 2 score (good RNA-Seq support) but a BLAST alignment indicating truncation could be scored as $2 \mathrm{P}$ with the direction of truncation indicated by using a 5 or 3 suffix, for example a 5 prime truncation would be scored ' $2 \mathrm{P} 5$ '.

\section{Comparison of the Red5 gene set with the 'Hongyang' gene annotation}

The manually annotated gene set was compared with the 39,040 published 'Hongyang' gene models originally published [14] (hereafter termed original 'Hongyang' model set) as well as to the 39,761 revised gene annotations [18] (hereafter termed revised 'Hongyang' model set). As 'Hongyang' is a different cultivar, polymorphisms are expected. To get a more accurate comparison, predicted protein sequences were used. When Red5 was used as query against the original 'Hongyang' model set as a database it was found that only $1973(\sim 6 \%)$ of the protein sequences for the 33,123 isoforms were identical in sequence and length to a 'Hongyang' predicted protein model [14]. We also detected instances where a Red5 model was perfectly contained within a 'Hongyang' model suggesting either the Red5 model is truncated, the 'Hongyang' model is over predicted or there is a genotypic difference between the two genotypes. The reverse situation where a 'Hongyang' model was perfectly contained in a Red5 model was also encountered. 882 $(2.67 \%)$ of Red5 proteins were perfectly contained within a longer sequence of an original 'Hongyang' model while $828(2.51 \%)$ Red5 proteins perfectly contained the sequence of an original 'Hongyang' model. When the revised 'Hongyang' model set [18] was employed as the database 3114 (9.4\%) Red5 protein sequences were found to be identical in sequence and length to a protein within the revised 'Hongyang' model set while 927 and 1007 Red5 proteins respectively either were perfectly encapsulated within a revised 'Hongyang' model or perfectly encapsulated a revised 'Hongyang' model. We repeated the analysis in the reverse direction. When using Red5 as the database, $42 \%$ of the original 'Hongyang' model proteins and $54.2 \%$ of the revised 'Hongyang' model proteins possessed a match with identity of $90 \%$ or greater, showing a considerable number of genes have been changed firstly in the revised annotation and secondly in the manual annotation process.

Comparison of the Red5 to original 'Hongyang' models identified 1958 original 'Hongyang' models had identical sequence and identical length to the corresponding Red5 model. A further 1261 original 'Hongyang' models possessed identical protein sequence to a Red5 model but were shorter in length than the Red5 model while for 576 original 'Hongyang' models the reverse was true. As expected 3114 of the revised models were found to have identical sequence and identical length to the corresponding Red5 model. A further 1685 revised 'Hongyang' models possessed identical protein sequence to a Red5 model but were shorter in length compared to the Red5 model while for 553 revised 'Hongyang' models the reverse was true. To examine the relationship with less than perfect matching best reciprocal BLASTP matches between Red5 and 'Hongyang' protein datasets identified for 19,179 proteins [14] and 21,479 proteins [18]. When the lengths of predicted proteins identified as best reciprocal BLASTp matches were compared, 5542 and 4700 proteins respectively within original and revised 'Hongyang' genes, respectively, possessed a longer protein sequence length than the Red5 model. By comparison 13,482 and 16,551 Red5 protein were longer than their best reciprocal BLASTp match counterparts from original and revised 'Hongyang' model sets respectively.

Within both the original and revised 'Hongyang' gene sets 148 and 113 models were completely missing from the Red whole genome sequence. The identifiers for these models are listed in Additional file 5. For 1195 original and 587 revised 'Hongyang' models lacking a best reciprocal BLASTp match we found the CDS for these models to be encapsulated in the UTR regions of Red5 models. For a further 379 original and 82 revised 'Hongyang' models lacking protein:protein matches to Red5 proteins we found their CDS to overlap the 3 UTR of a Red 5 model while for 362 original and 77 revised 'Hongyang' models the CDS was found to overlap the 5 UTR of a Red5 model. A further 3534 and 2034 models from the original and revised 'Hongyang' sets respectively were completely present in the whole genome sequence of Red5 but possessed no protein match to a Red5 gene model and did not align to a UTR region of a Red5 model. To identify if these models were missing from our annotation set due to lack of support from RNA-Seq evidence we merged BAM files for RNA-Seq libraries previous aligned to the Red5 whole genome sequence for purposes of assisting manual annotation. The CDS for the 'Hongyang' gene set of Yue and colleagues [18] was aligned to the Red5 whole genome sequence using GMAP [31] (version 2017-06-20), the resultant GFF3 output converted to Simplified Annotation Format (SAF) and RNA-Seq read counts to these features extracted using featureCounts [32].

Of the 2034 revised 'Hongyang' models that perfectly aligned to the Red5 whole genome sequence but for which there was not protein match of any kind, 535 aligned to regions of the Red5 genome where there was no aligned RNA-Seq and thus would have been unlikely to be manually annotated as a result. To further examine 
the RNA-Seq alignment of the remaining 1499 revised models, the base coverage on each chromosome was extracted using bedtools genomecov (v2.21.0) [33]. A perl script was then used to convert genomecov's chromosome base by base coverage to a bitmap ( 0 for no coverage at base position, 1 for coverage) and an array of coverage values for each exon of each of the 'Hongyang' 1499 revised alignments models. These were filtered to identity models incomplete coverage and models with coverage across their entire match regions. Of the 1499 revised models examined 131 possessed exons which were not supported by Red5 RNA-Seq while a further 372 revised 'Hongyang' models possessed RNA-Seq coverage of less than 5 reads per base. Given that we used all Red5 RNA-Seq combined in this analysis while annotators examined evidence library by library it is possible that these were not annotated due to inconsistent coverage across evidence libraries. The remaining 1069 regions represented 967 individual revised models each with RNA-Seq coverage of greater than 5 reads per base. We have provided a list of those revised models in Additional file 6 including their locations in the Red5 whole genome sequence and the average number of RNA-Seq reads aligned on a per base in each CDS.

In order to further assess our manual annotation gene set relative to the existing gene sets for 'Hongyang' we compared each set with 812 , bidirectionally sequenced, cDNA clones generated from Hort16A cDNAs selected in the A. chinensis EST sequencing program [25] (Additional file 7). When these 812 cDNA sequences were aligned to the gene sets of Red5 and 'Hongyang' 12, 58, and 39 did not possess a match to any CDS within the Red5, original and revised 'Hongyang' gene sets respectively while 635, 465 and 510 cDNAs aligned to these gene sets, respectively with $60 \%$ or greater identity (Table 3a). The longest ORF for each of the 812 cDNAs was extracted and the protein set was further reduced to 550 sequences (Additional file 8) by culling any sequence not likely to encode a full length protein sequence based on comparison to NCBI RefSeq Plant (version 76). These 550 protein sequences were compared to protein predictions of both 'Hongyang' and Red5 models and the best match identified for each of the 550 test set proteins. Of the 550 proteins in the test set 3,12 , and 9 possessed no protein match in the Red5, original, and revised 'Hongyang' gene sets respectively (Table $3 \mathrm{~b}$ ) while 144,29 , and 51 proteins from the test set possessed $100 \%$ identity to a protein within the Red5, original, and revised 'Hongyang' gene sets respectively (Table 3c). The number of matches with identity of $95-99 \%$ in Red5 was double that for the original 'Hongyang' gene set (Table 3c) while it was almost $1.5 \times$ that for the revised 'Hongyang' gene
Table 3 Alignment of DNA for 812 bidirectionally sequenced Hort1A cDNA sequences (A) and 550 Hort16A predicted protein sequences ( $B \& C$ ) with the Red5, original 'Hongyang' [14], and revised 'Hongyang' [18] gene model sets

\begin{tabular}{|c|c|c|c|}
\hline & $\begin{array}{l}\text { Red5 } \\
\text { Models }\end{array}$ & $\begin{array}{l}\text { Original } \\
\text { Hongyang } \\
\text { Models }\end{array}$ & $\begin{array}{l}\text { Revised } \\
\text { Hongyang } \\
\text { Models }\end{array}$ \\
\hline \multicolumn{4}{|l|}{ A } \\
\hline $\begin{array}{l}\text { Hort16A cDNA with BLAT match } \\
\text { identity }{ }^{\mathrm{a}}>=60 \%\end{array}$ & 635 & 465 & 510 \\
\hline $\begin{array}{l}\text { Hort16A cDNA with BLAT match } \\
\text { identity }^{\mathrm{a}}<60 \%\end{array}$ & 165 & 289 & 263 \\
\hline \multirow[t]{2}{*}{ Hort16A cDNA with no match } & 12 & 58 & 39 \\
\hline & 812 & 812 & 812 \\
\hline \multicolumn{4}{|l|}{ B } \\
\hline $\begin{array}{l}\text { Hort16A predicted protein with BLAT } \\
\text { match identity }{ }^{\mathrm{a}}>=60 \%\end{array}$ & 541 & 462 & 481 \\
\hline $\begin{array}{l}\text { Hort16A predicted protein with BLAT } \\
\text { match identity }{ }^{\mathrm{a}}<60 \%\end{array}$ & 6 & 76 & 60 \\
\hline \multirow{2}{*}{$\begin{array}{l}\text { Hort } 16 \text { A predicted protein with no } \\
\text { match }\end{array}$} & 3 & 12 & 9 \\
\hline & 550 & 550 & 550 \\
\hline \multicolumn{4}{|l|}{ C } \\
\hline \multicolumn{4}{|l|}{$\%$ Identity Range ${ }^{a}$} \\
\hline $0-59$ & 6 & 76 & 60 \\
\hline $60-64$ & 2 & 23 & 7 \\
\hline $65-69$ & 2 & 21 & 15 \\
\hline $70-74$ & 5 & 20 & 16 \\
\hline $75-79$ & 2 & 36 & 19 \\
\hline $80-84$ & 13 & 39 & 28 \\
\hline $85-89$ & 17 & 54 & 47 \\
\hline $90-94$ & 17 & 72 & 67 \\
\hline $95-99$ & 339 & 168 & 231 \\
\hline 100 & 144 & 29 & 51 \\
\hline
\end{tabular}

${ }^{a} \%$ identity was calculated as the number of matched bases (A) or matched amino acids ( $B$ \& $C$ ) between Hort16A and the named gene set divided by the total length of the Hort16A cds sequence $(A)$ or predicted protein sequence ( $B$ \& $C$ )

set. Collectively these results indicate that our manual annotation has yielded a gene set considerably different from previous gene sets for 'Hongyang' and while the revised gene set of Yue and colleagues [18] is a vast improvement over that of Huang and colleagues [14] our analyses suggest that our manual curation has further improved the overall structure of the $A$. chinensis gene set.

Using the new manually annotated gene models, two gene families were investigated further to assess whether new annotation had missed any genes. Given the reported poor annotation of EXPANSIN (EXP)-like genes [17], the genome sequence was translated into all six translation frames and used to identify regions that had 
homology to Arabidopsis EXP, and EXPANSIN-LIKE (EXPL) protein sequences. Fifty three chromosomal regions were identified, and 41 of these had a new manually annotated gene model. Of the 12 remaining gene models, six were partial regions of homology from which no gene models could be generated. Two regions each coded for a possible full length gene even though there was no associated computer-predicted model and no RNA-Seq evidence to support them. These two genes were scored Q0 and added to the gene list, producing a possible 47 EXP genes. Comparison of these 47 EXP genes with the 'Hongyang' gene models showed that six were identical to the 'Hongyang' gene models (Fig. 5a, Additional file 9), 18 were partially supported by an original 'Hongyang' gene model and 15 by a revised 'Hongyang' gene model. 29-32 EXP models were new and interestingly the majority of these were EXP genes with the EXPL more accurately predicted. A second gene family, the ACC SYNTHASE (ACS) genes, was also investigated [34]. In total 16 translated chromosomal regions showed homology to ACS proteins. Of these 16, 14 had a manually annotated gene model associated with them (Fig. 5b, Additional file 9). For the two regions that did not have a manually assigned gene, no convincing gene models could be derived. Two genes, ACS12 and $A C S 13$, had duplicate gene models that were $100 \%$ identical to each of the models at the nucleotide level, with ACS12 and ACS12R on two separate scaffolds on the unassigned chromosome (Chr30), suggesting these are possibly allelic. The other pair (ACS13 and ACS13R) were found as a tandem duplication on Chr12. When the 14 $A C S$ genes were compared with the original 'Hongyang' gene models, the 12 unique genes were all found; nine of these models were identical to the manual annotation, and three had a single exon/intron boundary difference, these numbers were unchanged in the revised 'Hongyang' models. The EXP study suggests that it is likely, even after manual annotation, that there are still unannotated genes of other gene families in the genome, especially in gene families of similar structure to the EXP genes i.e. smaller and computationally hard to predict. However, the new manually annotated genes do contain a more comprehensive list than the previous computationally generated gene lists.

At a chromosomal level, the gene density along the chromosomes varied, with less well populated regions associated with lower recombination rates (Fig. 6a). These lower density regions have been previously linked to centromeric regions $[35,36]$, and are also often associated with the translocation cut sites, consistent with the Robertsonian translocation. Especially clear are translocations of Chr1 to Chr9 and Chr8, where estimations of centromere locations can be made based on gene density (Fig. 6b). In other chromosomes such as Chr6 it is less clear, suggesting that within the same chromosome there are a number of translocation events that have occurred. Fig. $6 \mathrm{c}$ demonstrates that these other non-Robertsonian translocations are evident in Chr19, where low gene density regions flank a region of homology to Chr6 with a high gene density (Fig. 6c). As well as a WGD and subsequent translocations there are a considerable number of localised duplicated regions. Indeed, 1572 sites in the genome contained tandemly duplicated genes, representing $9.43 \%$ of annotated genes.

\section{Discussion}

In this project we have developed a whole genome sequence of a second genotype of $A$. chinensis genome which, in terms of assignment of scaffolds to pseudochromosomes, assigns all but $\sim 1 \%$ of assembled scaffolds to a linkage group. We also have a high quality dataset of annotated gene models using manual annotation. A more coherent genome will facilitate gene identification studies in segregating populations, and allow more accurate identification of QTLs to genomic regions and ultimately polymorphisms associated with the QTLs. Our genome incorporates $\sim 73 \%$ of the estimated genome size and to improve upon that further will likely require use of long read sequence technologies. The improved gene models will greatly enhance our molecular understanding of kiwifruit and plants in general, contributing a high-quality plant gene set for the plant community for global comparisons and underpinning molecular biology in this species.

During revision of the original 'Hongyang' annotation, Yue and colleagues [18] adopted the identifier format "AchXXgXXXXXX" where 'Ach' is the abbreviation of kiwifruit species name of $A$. chinensis in three characters. The two digits of a number following 'Ach' denoted the chromosome, and the next letter ' $\mathrm{g}$ ' identified the putative gene. This naming schema follows that for Arabidopsis. During our analysis we identified 21,479 genes having a best reciprocal BLAST match between the DNA coding regions of our Red5 model and those described by Yue and colleagues [18]. However, for 5934 of these best reciprocal gene matches there was conflict in chromosome assignment, suggesting that this was not the best method to use for genomes that are still improving. To this end we chose not to give a chromosome location as part of a gene name. This also lays the ground for the possibility for pan genome annotation within Actinidia species, it will accommodate further genome improvements, the issue of polyploidy, and possibility of chromosomal rearrangements that may have occurred in other Actinidia species. Finally given the heterozygosity of Actinidia species, and no current reference cultivar, we feel this naming methodology is currently the best option. 
a

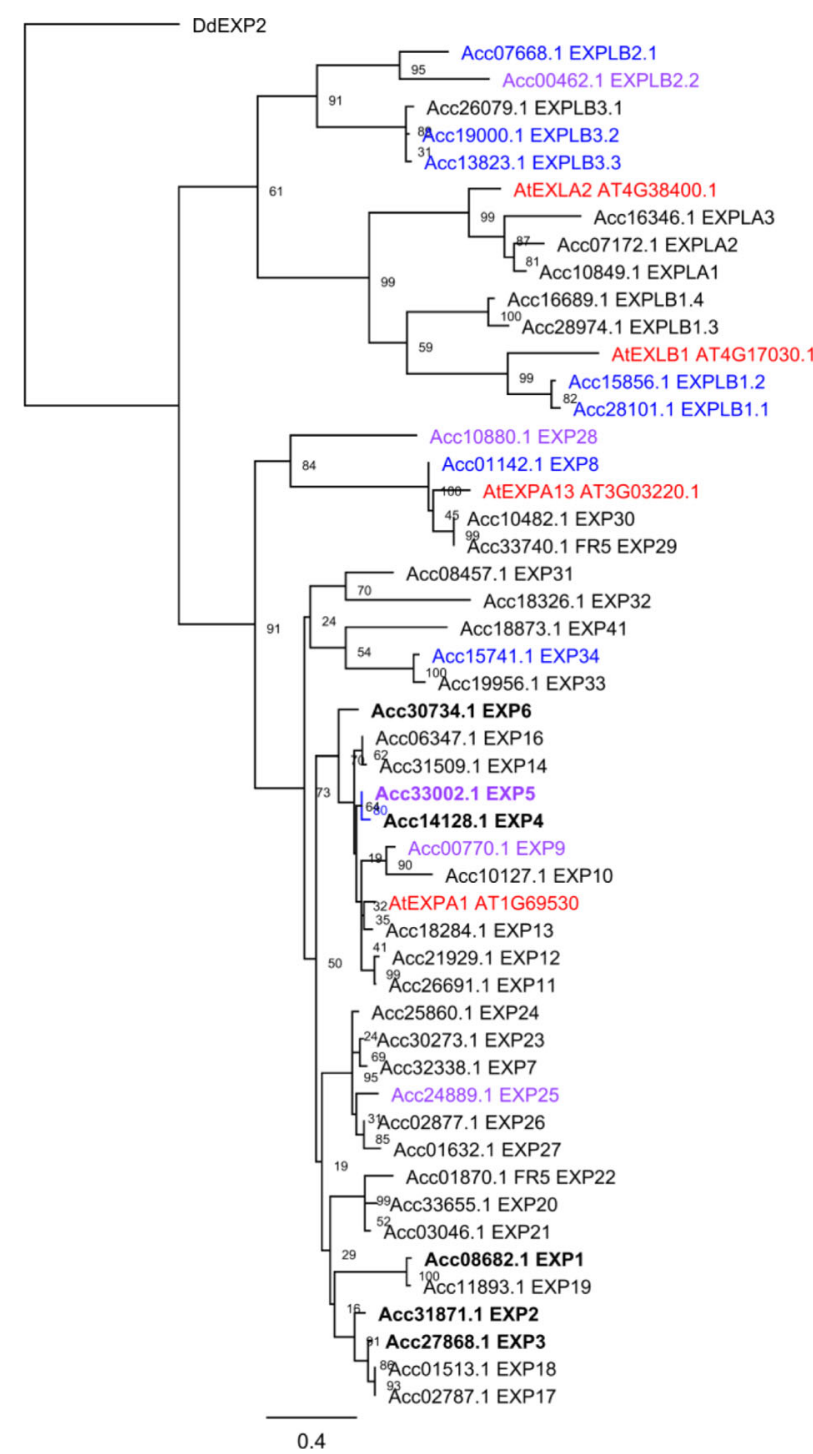

b

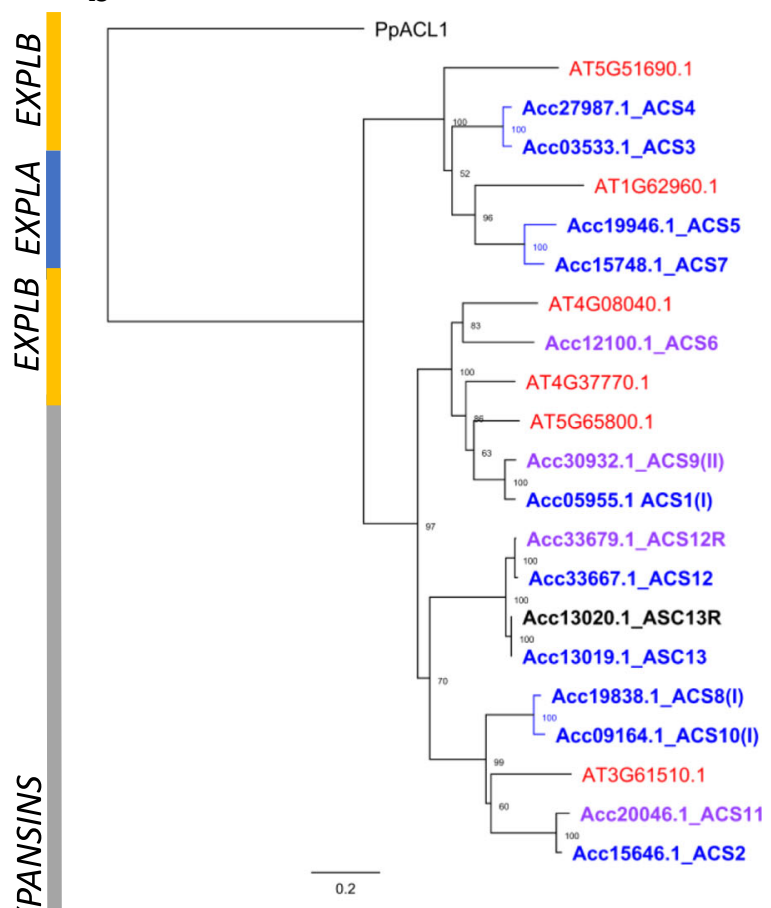

Fig. 5 Phylogenetic alignment of alignable protein sequences from EXPANSIN and ACC SYNTHASE proteins. Proteins in red are reference Arabidopsis proteins; blue and purple are published Actinidia chinensis protein sequences (blue are correctly annotated published unnamed 'Hongyang' proteins represented by gene models, purple models are partially represented); black are models are unannotated in the 2 published 'Hongyang' protein sets. Each tree is rooted with a basal species. Bootstrap values of 1000 iterations are given. A. EXPANSIN proteins; vertical line represents EXPANSIN proteins (Grey) EXPANSIN LIKE B proteins (Yellow) and EXPANSIN LIKE A proteins (blue). Rooted with DdEXP2 (NCBI \# gi|74,854,151) B. ACC SYNTASE Proteins. Rooted with PpACL1 (NCBI\# EDQ51432.1)

The available gene predictions from 'Hongyang' included only the coding regions of loci. Our visual annotation of the ends of the genes with RNA-Seq data enabled the identification of the complete transcriptional cassette in many instances. The importance of UTRs in gene regulation is becoming more and more apparent; for example, -uORFs have recently been shown to control vitamin $C$ production in kiwifruit [37] and there is a link between introns in the 5' UTR and transcription abundance [38]. In the manually annotated models of Red5 we observed that many of the transcripts had introns in the UTR and often these appeared to be alternatively spliced. Because of the large numbers of potential alternate UTR splice sites, as well as the ambiguity as to whether these are allelic differences, the UTR splice variants have not been included in the manually annotated gene set. These should be investigated further by other researchers when analysing individual genes.

We observed significant discrepancies between both the computer-predicted gene sets for A. chinensis 'Hongyang' and the manually annotated models for Red5. The number of changes was considerably greater 


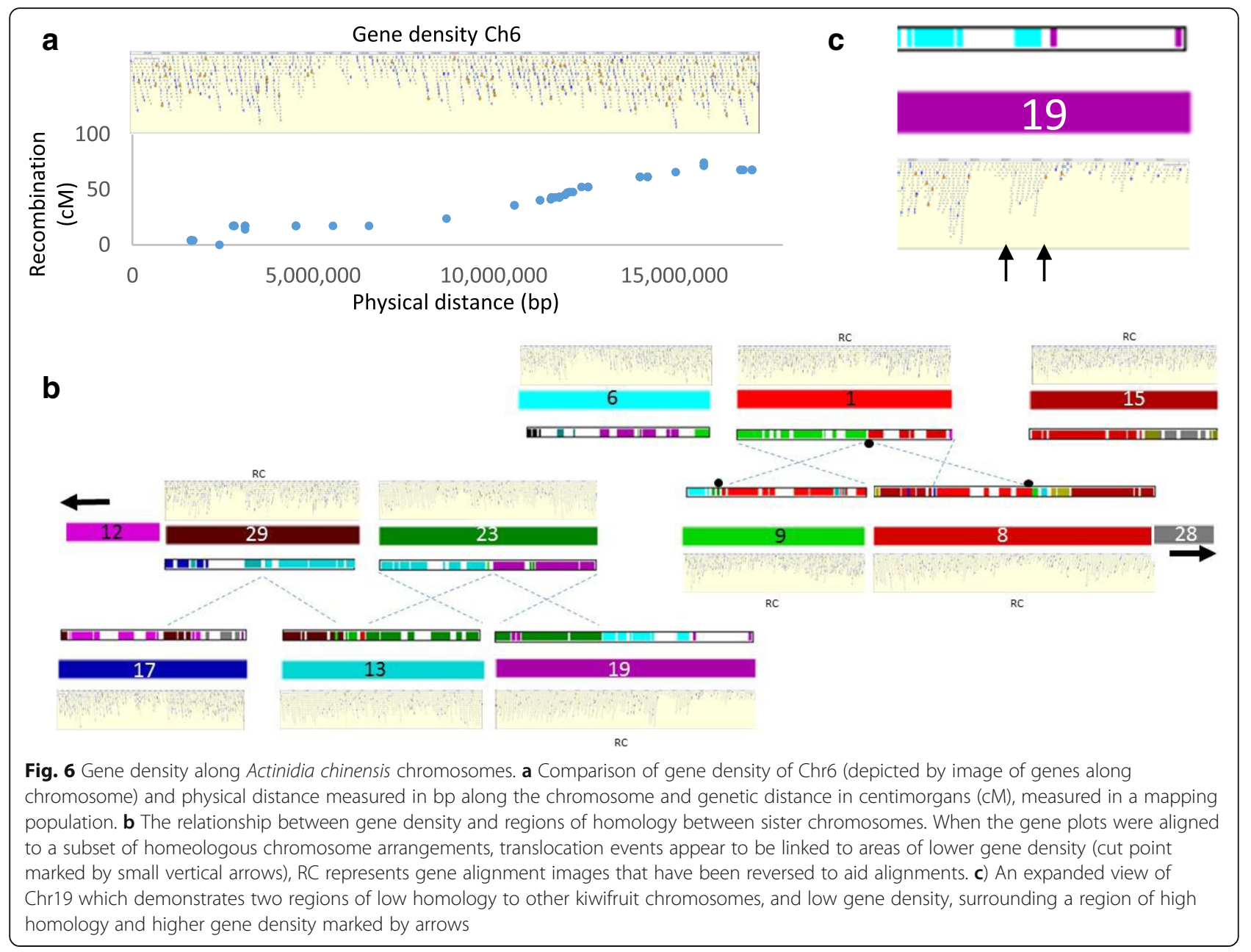

than for the recent re-annotated gene models in the Arabidopsis gene set [19] and this degree of difference was not expected given how closely related 'Hongyang' and Red5 are at the genome sequence level. While Arabidopsis is probably the best plant gene set in terms of quality of models, the recent improved annotation process still corrected $10 \%$ of the gene models [19]. The two examples of gene families given in this paper (Fig. 5) are the extreme examples of computer prediction, the ACS genes that were accurately predicted and the EXP genes that were very poorly predicted. This annotation methodology needs to be optimised by each gene family showing a complexity required in building automated annotation pipelines. An issue noted during our study was that the increased speed of annotation conferred by having a large number of annotators was balanced by inconsistent interpretation of gene structure by individual annotators, particularly for models that were harder to interpret. The scale of these inconsistencies meant that it was necessary for a small number of 'expert' annotators to check each gene model (Additional file 10), adding a considerable time to the project but greatly increasing the quality of the output. The manual annotation inconsistencies were almost always around the harder to annotate (Q1) gene models, with Q2 gene models usually not needing to be adjusted. This variation among manual annotators could be addressed by more clearly setting out expectations of how to deal with conflicting data during pre-annotation training.

Our development of gene models was weighted heavily towards the use of RNA-Seq evidence, together with indications from the computational gene models. However, the RNA-Seq did not always give a clear picture of gene structure. Very often there were reads that mapped to intronic regions; sometimes these reads were specific to a single intron (Fig. 4a), and sometimes they were distributed across the whole gene (Fig. 4b). Sometimes these anomalies could be resolved using transcription data from different RNA-Seq libraries (Fig. 4c). The reads were assumed to either be part of intron readthrough, or antisense transcription associated with those genes [39]. The piecing together of RNA-Seq reads in intergenic regions with no apparent open reading frame associated with them is often ambiguous. While these 
may be associated with non-coding RNA and they are currently left unannotated (Fig. 4d).

WGD has been proposed as promoting diversification of gene function [40]. Within the $A$. chinensis genome there is also evidence of global duplication, as well as local gene duplications. The global changes caused by Robertsonian chromosomal translocations noted in our genome assembly were originally identified in animals and often lead to birth defects. In a duplicated plant genome any such effects of these changes must be buffered and indeed have been previously reported in polyploid plants such as wheat [41], Brassica oleracea [42] and strawberry [43]. The duplication has caused previously identified single copy genes [44] (Table 4) to exist as two or more copies in the Red5 genome, with the exception of the three which appeared only to have a single copy. Many of the duplicates of the single copy genes were found on the respective homeologous chromosomes, as shown in bold in Table 4. The loss of function of a duplicated gene was sometimes observed with transcribed genes with no open reading frame (such as Chr1, position $10.85 \mathrm{Mb}$ has a functional homologue on Chr9 (Acc09963)).

\section{Conclusions}

Our study provides a second genome with a high quality gene set to the kiwifruit research community, and we are confident that the 27,783 genes with a Q2 score are good quality gene models representing transcribed genes. When these are used for comparative purposes in analyses of other plant genomes, either through computational prediction or through addition of tracks for manual assignment, our models will provide users with greater confidence in their newly developed gene models. Our work highlights that a labour-intensive human intervention is still the most accurate way of predicting genes, and identifies improvements that need to be made in computational predictions of coding sequences and intron/exon boundaries.

\section{Methods}

\section{Plant material}

Two $\mathrm{F}_{1}$ diploid $A$. chinensis Planch. var. chinensis from an open pollinated red-fleshed fruiting mother were screened with 8 microsatellites (Additional file 11) to ascertain that they were true siblings. These were crossed and $F_{2}$ offspring, a female with red fruit (Red Female 2) and male were selected for further crossing. Forty $F_{3}$ progeny were sown, with each having an inbreeding coefficient of 0.375 (Fig. 1). A red fruiting female (Red5) was chosen for genome sequencing. For gene expression analysis (RNA-Seq), different tissues were harvested from mature Red5 plants to encapsulate a diversity of expression (Table 3).

\section{DNA isolation and sequencing}

Nuclear DNA was isolated from leaf tissue of Red5 using nuclei enrichment and DNA extraction as described by

Table 4 Analysis of single copy genes in the Actinidia chinensis genome

\begin{tabular}{|c|c|c|c|c|c|c|c|}
\hline \multirow[t]{2}{*}{ Arabidopsis } & & \multicolumn{6}{|l|}{ Kiwifruit } \\
\hline & & Best hit 1 & Chr & Best hit 2 & Chr & Best hit 3 & Chr \\
\hline AGT1 & AT2G13360 & Acc30351.1 & 26 & Acc32422.1 & 28 & & \\
\hline MAG1 & At3g47810 & Acc29850.1 & 26 & Acc04108.1 & 3 & Acc02358.1 & 2 \\
\hline DIENELACTONE HYDROLASE- like & At2g32520 & Acc13638.1 & 12 & & & & \\
\hline ATPQ & At3g52300 & Acc25925.1 & 23 & Асc30188.1 & 26 & Acc32243.1 & 28 \\
\hline RIBOSOMAL PROTEIN SBe & At5g06360 & Acc12882.1 & 11 & Acc17591.1 & 16 & & \\
\hline RRM & At5g04600 & Acc04134.1 & 3 & Acc27568.1 & 24 & & \\
\hline MGP1 & At2g21870 & Acc05500.1 & 5 & Acc10590.1 & 9 & & \\
\hline EIF3K & At4g33250 & Acc12920.1 & 12 & Acc23419.1 & 20 & & \\
\hline Fb15 & At4g30010 & Acc12893.1 & 11 & Acc17577.1 & 16 & Acc27046.1 & 23 \\
\hline CCP2 & At1g77710 & Acc05095.1 & 4 & Acc24370.1 & 21 & & \\
\hline Glycine rich protein & At4g08230 & Acc05092.1 & 4 & & & & \\
\hline Cytochrome c oxidase & At4g37830 & Acc05943.1 & 5 & Асc30914.1 & 27 & & \\
\hline Unknown & At5g47570 & Acc16748.1 & 15 & Acc15793.1 & 14 & Acc19901.1 & 18 \\
\hline PFD5 & At5g23290 & Acc28316.1 & 25 & Acc31379.1 & 27 & & \\
\hline Unknown & At1g27530 & Acc04831.1 & 4 & Acc23863.1 & 21 & & \\
\hline RIDA & At3g20390 & Acc21627 & 19 & & & & \\
\hline Unknown & At5g63135 & Acc22027.1 & 19 & Acc26769.1 & 23 & & \\
\hline
\end{tabular}

Best BLAST hits to the manually annotated gene models. Chromosome numbers in bold indicates the predicted homeologous chromosomes 
Naim and colleagues [45]. DNA was sheared to an insert size of either $\sim 160 \mathrm{bp}$ or $\sim 240 \mathrm{bp}$ and prepared for 100 base paired-end sequencing along with 100 base longinsert mate-paired-end (LIMP) libraries with average insert sizes of 4, 9 and $13 \mathrm{~Kb}$ and sequenced on Illumina HiSeq2000 ${ }^{\text {ma }}$ (Illumina Inc. San Diego, CA, USA) at the Australian Genome Research Facility (AGRF - Brisbane), according to the manufacturer's instructions. A $4 \mathrm{~Kb}$ insert library was also prepared for paired-end sequencing by Life Science (Roche) 454 GS-FLX. Cyclically corrected sequences from a small number (6) of PacBIO SMRT cells (45 $\mathrm{Mb}$ per cell) were also included during gap closing.

\section{RNA isolation and sequencing}

RNA was extracted using the method described in Chang and colleagues [46]. RNA samples were quantified and sample purity was verified by using a Nanodrop ND-1000 spectrophotometer (Thermo Fisher Scientific). RNA integrity was checked by an Agilent 2100 Bioanalyzer (Agilent Technologies, Palo Alto, CA, USA). RNA was supplied to Macrogen Inc. (Seoul, South Korea) for standard RNA-Seq preparation and sequenced using the Illumina HiSeq $2000^{\text {tw }}$ yielding either single or pair-end RNA-Seq reads.

\section{Bacterial artificial chromosome (BAC) library sequencing} A total of 11,520 clones from a BAC library made from nuclear DNA of the F1 mother (Red Female 1) were selected for sequencing. The re-arrayed BAC clones were grown in 96-well plates containing $1.2 \mathrm{~mL} \mathrm{LB}$ liquid medium with $12.5 \mu \mathrm{g} / \mathrm{mL}$ tetracycline at $37{ }^{\circ} \mathrm{C}$ in an orbital shaker at $180 \mathrm{rpm}$ for $16 \mathrm{~h}$. The bacterial cells were harvested at $3000 \mathrm{rpm}$ at room temperature for $30 \mathrm{~min}$ in a benchtop centrifuge. The BAC DNA was extracted using a plate based alkaline lysis method [47] and dissolved in $150 \mu \mathrm{L} 28 \mathrm{mM}$ Tris- $\mathrm{HCl} \mathrm{pH} 8,1 \mathrm{mM}$ EDTA, $0.6 \mathrm{mM}$ cresol red (to provide a visual aid for robotic transfers). Each BAC was individually barcoded using an in-house method BACRB (details of which can be supplied on request to the corresponding author). The barcoding oligonucleotides (BioSearch Technologies, Novato, CA, USA) were dissolved in TE buffer $(10 \mathrm{mM}$ Tris- $\mathrm{HCl} \mathrm{pH}$ 7.5, $1 \mathrm{mM}$ EDTA) to a final concentration of $50 \mathrm{pmol} / \mu \mathrm{L}$. Approximately $15 \mathrm{ng}$ of BAC plasmid $(5 \mu \mathrm{L})$ was randomly tagged by primer extension preamplification PCR (PEP-PCR) in $50-\mu \mathrm{L}$ reactions using AccuPrime $^{\text {Tn }}$ Taq DNA polymerase High Fidelity system as per the manufacturer's instructions (Life Technologies Corporation, Carlsbad, CA, USA) and 10 pmol of a corresponding BACRB oligonucleotide. The DNA was denatured at $94{ }^{\circ} \mathrm{C}$ for 2 min and amplified using 50 cycles of: $94{ }^{\circ} \mathrm{C}$ for $40 \mathrm{~s}$, a two-step annealing strategy $\left(30{ }^{\circ} \mathrm{C}\right.$ for $2 \mathrm{~min}$, ramp at $0.1{ }^{\circ} \mathrm{C} / \mathrm{s}, 48{ }^{\circ} \mathrm{C}$ for $\left.4 \mathrm{~min}\right), 68{ }^{\circ} \mathrm{C} 60 \mathrm{~s}$, followed by a final extension at $68{ }^{\circ} \mathrm{C}$ for $7 \mathrm{~min}$. The randomly tagged $\mathrm{BAC}$ amplicons of each clone were amplified with the tailed BACRB oligonucleotide in a second touchdown (TD) amplification process. One microlitre of randomly tagged $\mathrm{BAC}$ amplicon was added to $19 \mu \mathrm{L}$ of PCR mix $(0.6 \mathrm{M}$ trehalose, $40 \mathrm{mM}$ Tris- $\mathrm{HCl}$ $\mathrm{pH} 8,20 \mathrm{mM} \mathrm{KCl}, 20 \mathrm{mM}\left(\mathrm{NH}_{4}\right) 2 \mathrm{SO}_{4}, 10 \mu \mathrm{g}$ BSA, 0 . $5 \mathrm{mM} \mathrm{MgSO}, 0.2 \mathrm{mM}$ dNTP, $2 \mathrm{pM}$ tailed-BACRB oligonucleotide, and 0.25 unit Platinum ${ }^{\circ} \mathrm{Pfx}$ DNA polymerase (Life Technologies)). The touchdown amplification was performed as follows: $94{ }^{\circ} \mathrm{C}$ for $2 \mathrm{~min}, 1$ cycle; $\left(94{ }^{\circ} \mathrm{C}\right.$ for 30 s, TD $60-50{ }^{\circ} \mathrm{C}$ for $30 \mathrm{~s}, 68{ }^{\circ} \mathrm{C}$ for $1 \mathrm{~min}$ ), 20 cycles; $\left(94{ }^{\circ} \mathrm{C}\right.$ for $30 \mathrm{~s}, 50{ }^{\circ} \mathrm{C}$ for $30 \mathrm{~s}, 68^{\circ} \mathrm{C}$ for $1 \mathrm{~min}$ ), 10 cycles; and final extension at $68{ }^{\circ} \mathrm{C}$ for $7 \mathrm{~min}$. The barcoded samples from each 384-well plate were pooled, concentrated, and analysed by agarose gel electrophoresis. A barcoded TruSeq library was prepared for each plate pool (30 barcoded libraries). A super pool was prepared by combining 10 barcoded libraries of plate pools. A total of three super pools were obtained, and each one sequenced separate lanes on single end mode, at Macrogen Inc. (Seoul, South Korea). The three lanes generated 576.35 million reads, comprising $58.2 \mathrm{Gbp}$.

For Roche 454 GS-FLX sequencing, 50-mL cultures were grown and extracted as described above. The DNA pellet was dissolved in $50 \mu \mathrm{L}$ buffer $(10 \mathrm{mM}$ Tris- $\mathrm{HCl}$, 1 mM EDTA, pH 7.5) and sent to be sequenced by 454 GS-FLX at Macrogen Inc. (Seoul, South Korea).

\section{Assembly}

A "PseudoSanger"-like approach [26] was used to assemble two paired-end read libraries with stepwise decreasing insert size (240 and 160 bases, respectively). The libraries yielded 169,008,438 and 170,367,691 read pairs, respectively. Prior to assembly, reads were errorcorrected using the error correction tool from the ALLPATHS-lg assembler [48] yielding 159,232,897 and $167,054,602$ corrected read pairs, respectively. These reads were also used to estimate genome size using both preQC (https://github.com/jts/sga/wiki/preqc) from sga [49] and jellyfish (version 1.1.10) [50]. Error-corrected reads were assembled using anytag (version 2.5.2) [26]. Anytag yielded 46,117,212 fragments with a minimum length of 81 bases, maximum length of 450 bases and N50 of 275 bases. These fragments were assembled using Newbler 2.9 (Roche 454, Bradford, Connecticut, USA) with settings "-m -large -het -cpu 32". Also included in the Newbler assembly were 1,209,245 paired end sequences from a 4-kb insert library sequenced using 454 GS-FLX pyrosequencing. UniVec_Core (ftp://ftp.ncbi. nlm.nih.gov/pub/UniVec/UniVec_Core) and common sequencing primers were used for vector trimming during Newbler assembly, while the sequence of Escherichia coli DH10B (NC_010473.1) was used for screening for bacterial contamination. 
Reads from Illumina LIMP libraries were trimmed to 36 bases and redundant pairs removed prior to use using a custom perl script. The resulting read pairs were then used to scaffold the Newbler assembly further using SSPACE2 [27], which was then followed by two iterations of gap closure using GapCloser (v1.12; http://soap.genomics.org.cn/about.html). Two additional Illumina paired-end read libraries, not used in the anytag/Newbler-based contig assembly, were also employed during gap closure along with the assembly input read libraries. These two additional libraries possessed average insert sizes of $\sim 240 \mathrm{bp}$ and $\sim 300 \mathrm{bp}$ with read lengths of $75 \mathrm{bp}$ (trimmed to 64 bases) and $150 \mathrm{bp}$ (trimmed to 109 bases) respectively. Gap closure using Illumina-derived sequence reads yielded a reduction in ambiguities $(\mathrm{N})$ within the assembly from 17.3 to 3 . $57 \%$. Cyclically corrected sequences extracted from 6 SMRT $^{\mathrm{Th}}$ cells (PacBIO) containing 35-45 Mb per cell were compared with the assembly using BLAT [51] and the resulting alignments used to further infill $199.01 \mathrm{~Kb}$ of genome assembly gaps. This assembly process yielded 3387 scaffolds containing $554 \mathrm{Mb}$ with a minimum size of 1997 bases, a maximum size of 4,436,233 bases, a mean size of 142,646 bases, and N50 of 623,820 bases, an N90 of 140,742 and $3.54 \% \mathrm{~N}$.

Assembled scaffolds were joined using 100 base read data from the sequencing of 11,520 BAC clones. Unique BAC clone reads were extracted and mapped to the assembly scaffolds using megablast (-W 70) [52]. A custom perl script was used to filter out reads mapping at less than $100 \%$ of their length and then to merge scaffolds determined to be co-linear. The genetic map of Scaglione and colleagues [15] was used to guide assignment of the vast majority of scaffolds to linkage groups. A few remaining unassigned contigs were assigned to linkage groups using genetic markers from two other sources (Fraser and colleagues [28] and Additional file 12). To enable its use with Red5, markers were first converted to fasta sequences. For Single Nucleotide Polymorphisms (SNP) markers a sequence consisting of the SNP plus 500 upstream and downstream flanking bases was extracted from scaffolds of 'Hongyang' [14], grandparent assembly Red Female 1( $\mathrm{R}$ Crowhurst, unpublished), an unrelated yellow fleshed $A$. chinensis assembly CK15_02 (R Crowhurst, unpublished) or the Red5 assembly herein, as appropriate. For each SNP the extracted fasta was named so as to encode the scaffold of origin, the location of the SNP in the scaffold of origin, the sequence region extracted from the scaffold of origin, the linkage group and centimorgan position from the genetic map and the map of origin. The names of the fasta sequences as described are provided in Additional file 12. For EST-based markers
[25] the sequence of the EST was obtained from NCBI GenBank. The FASTA sequences for markers were aligned to assembly scaffolds for Red5 using megablast (-W 50) and filtered to remove alignments of less than $98 \%$ of overall length and identity before being used for assignment of scaffolds to linkage groups. An 'all by all' megablast comparison of scaffolds of 'Hongyang' [14] and Red5 was used to assign further Red5 scaffolds to linkage groups using BLAST walking from already assigned Red5 scaffolds. Red5 scaffolds identified as chimeric based on marker evidence (assigned to more than one linkage group or location within a linkage group) were manually inspected, split at identified break points and the component parts re-assigned as supported by evidence (Additional file 12).

To assess the level of DNA sequencing incorporation into the final assembly, the DNA sequencing libraries used as inputs to the anytag software were aligned to the chromosome assemblies using bowtie2 [53] using command line options: -end-to-end -very-fast -I 50 -X 500 -fr -threads 8. To enable comparison with 'Hongyang', mapping was repeated using the 'Hongyang' chromosomes [14] as the reference.

To assess the accuracy of the assembly, 22 clones from the BAC library of $A$. chinensis Red Female 1 were selected such that each contained sequence spanning two markers located on Chr25. DNA for each clone was individually barcoded and sequenced using the Life Sciences (Roche) 454 GS-FLX platform. The sequences were assembled using Newbler (version 2.9) and the assembled BAC contigs compared with the Red5 genome assembly using megablast with a word size set at 50 . Results were filtered to remove regions of repetitive sequence alignment or alignments with less than 98\% match to the Red5 genome sequence. Alignments were converted to GFF3 format and visualised using Geneious (versions 8.1.2) [54]. Additionally, reads from the $9 \mathrm{~Kb}$ LIMP library were aligned to individual chromosomes for the Red5 whole genome assembly using bowtie2 [53] using the following command line options: -end-to-end

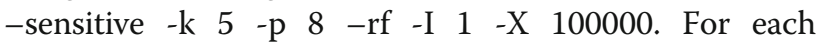
chromosome the distance between mate pairs was visualised using hagfish_blockplot from the software 'hagfish' (https://github.com/mfiers/hagfish/). Individual chromosome plots were then cut and pasted to form a montage. Each plot represents the alignment across the entire length of a chromosome. Plots were produced to a standard pixel width irrespective of chromosome length. Green regions indicate mate pairs aligning to the chromosomes within the expected distance for the library. Black indicates regions without mate pair alignment. Pinkish-red indicates regions where the distance between mated paired end reads is shorter (assembly 
compression relative to physical genome) or greater (assembly expansion relative to physical genome).

\section{Mapping of Red5 RNA-Seq libraries to assembly}

The RNA-Seq reads were mapped to the chromosome assemblies of Red5 using the STAR RNA-Seq aligner [55] (version STAR-STAR_2.4.2a) using the command line parameters "-chimSegmentMin 30 -runMode alignReads -alignIntronMin 21 -alignIntronMax 25000 -alignMatesGapMax 25000 -alignEndsType Local". All RNA-Seq reads were trimmed by 13 bases at the $5^{\prime}$ end prior to use. Reads were additionally trimmed at their $3^{\prime}$ ends when quality score assessment with FastQC (http://www.bioinformatics.babraham.ac.uk/projects/fastqc/) gave quality values below 20 .

\section{Gene annotation}

Manual curation of gene models was performed as follows. The published gene models for 'Hongyang' [14] and A. chinensis ESTs within NCBI GenBank were downloaded and mapped to the assembled pseudo-chromosome sequences of Red5 using GMAP (version 2014-10-22) [31]. The sequences of the Red5 pseudo-chromosomes were repeat masked using RepeatMasker (version open-4.0.5) with options "-e ncbi -pa 30 -s -nolow -species viridiplantae -a -x -poly -gff" and with the RepBase (http://www.girinst.org/) RepeatMasker libraries (20140131). Ab initio gene model prediction was performed using Augustus-3.1 [56] employing command line options "-species $=$ arabidopsis" and evidential hints from both RNA-Seq derived from Red5 (Table 3) and from 47,384 A chinensis EST sequences (downloaded from NCBI GenBank) using described protocols (http://bioinf.uni-greifswald.de/bioinf/wiki/pmwiki. $\mathrm{php} ? \mathrm{n}=$ Augustus.Augustus). The genome sequence, $\mathrm{ab}$ initio predicted models, mapped ESTs and 'Hongyang' gene models as well as RNA-Seq alignments were imported into WebApollo1 [21] and made available for community-based manual curation via Amazon Cloud. Where evidence suggested multiple isoforms that could clearly be defined each isoform was included. Where it was not possible to differentiate individual isoforms unambiguously a single model was submitted by community annotators. After initial annotation, all models were ported to WebApollo2 (version 2.0.2) (https:/github.com/ GMOD/Apollo/releases/tag/2.0.2) and each annotation was reviewed manually. This review was followed by computational analysis to identify anomalous annotations such as small $(<15 \mathrm{bp})$ introns and untranslated exons numbering more than 3 . These models were rechecked and often further modified or removed. The manually annotated gene models were named using the prefix Acc (for Actinidia chinensis var. chinensis) and sequentially numbered. The cDNA, CDS, peptide and GFF3 records for each model were committed into a GitHub repository to enable tracking of changes over time. A schema for manual annotation can be found in Additional file 10.

\section{Estimating genome completeness using BUSCO analysis}

Benchmarking Universal Single Copy Orthologs (BUSCO analysis) [30] was used to examine genome completeness. Version 2.0 of BUSCO was used with NCBI Blast+ version 2.2.30, AUGUSTUS version 3.2.2 [56] with "-species arabidopsis", HMMER version 3.1b2 (http://hmmer.org) and the Embryophyta_odb9 dataset (http://busco.ezlab.org/ datasets/embryophyta_odb9.tar.gz). BUSCO analysis was also undertaken on the 'Hongyang' genome [14].

\section{Comparisons of gene sets}

To further evaluate our manually annotated gene models we compared their coding regions with the sequence of 859 clones from cDNA libraries for $A$. chinensis var. chinensis 'Hort16A', within our in-house sequence database which had been previously individually cloned and DNA extracted [25]. The cDNA clones were bi-directionally sequenced in full, using 'Sanger' sequencing, and then sequences with greater than $98 \%$ similarity were removed using cd-hit-est [57], yielding a comparison set of 812 cDNA sequence with minimum, maximum, and mean lengths of 247, 4506 and 1495 bases respectively and an N50 of 1653 bases (Additional file 7). Coding regions of our manual annotation data set as well as those for 'Hongyang' were compared with these cDNA sequences using BLAT (version 36) and GMAP as follows. The longest ORF for each of the 812 cDNAs was extracted and sequences not likely encoding a full length protein based on comparison to NCBI RefSeq Plant (version 76) were removed leaving 550 proteins. Each of the 550 protein sequences was compared to the predicted protein sequences of the 3 gene sets (Red5, original and revised 'Hongyang') using BLAT and the best alignments summarised using a custom perl script.

To compare whole gene sets, for each gene set pair (Red5 with original 'Hongyang' and Red5 with revised 'Hongyang') the following analyses were undertaken: (1) predicted proteins of each pair of gene sets were compared to each other using BLAT, (2) CDS sequences for each gene set pair were compared using BLAT, (3) the CDS were aligned to the genome sequence using GMAP (version 2017-06-20). A custom perl script was then used to summarise these analyses by first seeking the best protein:protein alignments, then the best CDS:CDS alignments and finally the alignment of the query to the whole genome sequence. The summarisation perl script takes into account query and target sequence lengths for protein:protein and CDS:CDS alignments as well as genome alignment co-ordinates from the GMAP alignments in order to yield the metrics for best alignments including: number of alignments assigned to bins based on 
percentage identity, the number showing a length variance (query equal/shorter/longer than target), number of queries encapsulated with the UTR of a target gene model, number of queries overlapping the 5 or 3 UTR of a target gene model and the number missing from results files for these analyses. Additional file 13 summarises the raw results for the best BLAT gene set match to each cDNA protein. BLAT results presented in Additional file 13 were extracted using "awk ' Percent $=(\$ 1 / \$ 11) * 100$; print $\$ 10 "\left|t " \$ 11^{\prime \prime}\right| t " \$ 1 " \mid t "$ Percent"|t"\$14"|t"\$15\}" then the difference in length between summary data columns 2 and 6 added in Microsoft Excel.

\section{Phylogenetic analysis}

Gene models were selected and aligned in Geneious (R10.0.3) (www.geneious.com) using Geneious Alignment (with free end gaps), Gap opening penalty 30, extension penalty 0 and refinement iterations 2 . Alignable regions were extracted, realigned and clustered using PHYML [58] default settings. Data from 1000 bootstrap sets are presented.

\section{Additional files}

Additional file 1: Map back rates to the Red5 genome

sequence.Summary of the numbers of input reads reads that align to the RED5 genome construction (XLSX $10 \mathrm{~kb}$ )

Additional file 2: BAC alignment to chromosome 25.Table summarising alignments of contigs from 22 BAC clones to chromosome 25 of the Red5 assembly (XLSX $15 \mathrm{~kb}$ )

Additional file 3: Comparison of predicted paired end distance to genome.Heatmaps of alignment distance scores for the alignment of the read pairs from the 9Kb long-insert mate-paired-end (LIMP) library to each of the 29 chromosomes within the Red5 whole genome assembly and. Individual chromosome plots were prepared using hagfish_blockplot from the software program 'hagfish' (https://github.com/mfiers/hagfish/). Individual images were cropped for height (not length) then cut and pasted into a table format for easier viewing. Each image depicted the entire length of the chromosome but all images are of standard length irrespective of chromosome length. Green regions indicate mate pairs aligning to the whole genome sequence within the expected distance of the library. Black indicates regions without mate pair alignment. Pinkishred indicates regions where the distance between mated paired end reads is shorter (assembly compression relative to physical genome) or longer (assembly expansion relative to physical genome). (PPTX $432 \mathrm{~kb}$ )

Additional file 4: BLASTP comparison of manually edited gene models to the revised 'Hongyang' gene models. List of best reciprocal BLASTp matches between the revised Actinidia chinensis 'Hongyang' genes [18]and the Red5 gene set (XLSX $436 \mathrm{~kb})$

Additional file 5: 'Hongyang' Gene models that align to Red5 genome.List of Actinidia chinensis 'Hongyang' genes that align to the Red5 whole genome sequence. Additional file 5A: models from original 'Hongyang' annotation [14]. Additional file 5B: models from revised 'Hongyang' annotation [18] (XLSX 19 kb)

Additional file 6: Revised 'Hongyang' genes omitted from the manually edited gene set. Average RNA-Seq read coverage of the 1069 KIR V2 models perfectly aligned to the Red5 genome without a protein match in the Red5 gene set. (XLSX $114 \mathrm{~kb}$ )

Additional file 7: Details of sequenced cDNA's generated. Fasta formatted sequences of 812 bidirectionally sequenced expressed sequence tag clones from $A$. chinensis var. chinensis used in evaluating manually annotated gene models of Red5. (FASTA $1204 \mathrm{~kb}$ )

Additional file 8: Sequenced CDNA's used to verify the gene models.Fasta formatted predicted protein sequences of 550 bidirectionally sequenced expressed sequence tag clones from $A$. chinensis var. chinensis used in evaluating manually annotated gene models of Red5. (FASTA $220 \mathrm{~kb}$ )

Additional file 9: Selected gene models used for phylogeny.Table of EXPANSIN genes and ACS genes identified in this study (XLSX 19 kb)

Additional file 10: The manual annotation process.Flow diagram of manual annotation process. A. Timeline showing the manual annotation process. *see materials and methods. B. Annotation followed a 5 step process. The annotator training was completed in the form of both workshops and YouTube training videos. **https://www.youtube.com/ playlist?list=PLcBe8nhQVgUg1zqOsdeRuVq9QVsLfj_Y9. (PPTX 47 kb)

Additional file 11: Parental tests of the Red5 genotype. Results from $F_{1}$ Sibling test for the first cross. (DOCX $14 \mathrm{~kb}$ )

Additional file 12: Markers used in this study. List of names of extracted FASTA sequences for SNP markers used to construct the genome (TXT $841 \mathrm{~kb}$ )

Additional file 13: Comparison of Actinidia EST's to manually annotated gene models.Summary of the raw results for the best BLAT gene set match protein of the sequenced cDNAs and manually annotated predicted peptides. (XLSX $102 \mathrm{~kb}$ )

\section{Abbreviations}

ACS: ACC Syntase; BLAST: Basic Local Alignment Search Tool; BUSCO: Benchmarking Universal Single-Copy Orthologue; CDS: Coding Sequence; EST: Expressed Sequence Tag; EXP: Expansin; WGD: Whole genome duplication

\section{Acknowledgements}

We would like to thank Zespri for support of this project.

\section{Funding}

This work was funded by Kiwifruit Royalty Investment Program by The New Zealand Institute for Plant \& Food Research Ltd. with support from Zespri, and the CORE grant from the New Zealand Ministry of Business, Innovation and Employment (MBIE) (C\#27353) and its predecessors. The funding bodies had no role in the design of the study, the collection, analysis, or interpretation of data or writing this manuscript.

\section{Availability of data and materials}

This Whole Genome Shotgun project has been deposited at DDBJ/ENA/ GenBank (https://www.ncbi.nlm.nih.gov) under the accession NKQK00000000. The version described in this paper is version NKQK01000000. For community contributions please check the list of FASTA gene models available on the Plant and Food GitHub Repository (https://github.com/ PlantandFoodResearch/Red5_WGS_Manual_Annotation) and will be updated as the gene models are further refined.

\section{Authors' contributions}

The Red5 genome was initiated by RPH, RC, PD, EH. The genetic maps were constructed by DS, PD, LF, MM, SG, YL, QZ, NdS, HB, DC and RT. The genome consortium was initiated by HW RT RJS. DNA extraction and BAC libraries were made by EH ZL. Genome construction was undertaken by RC, with other bioinformatics support from HD, CD, JM, RS, AHT, ST, CD and MD. Manual annotation was undertaken by many of the authors detailed here and all the other authors in the authorship list. The manual annotation was led by RJS, SP, RC, and checked by SP, RC, SN, LF, YP, KG, RS, JT, SCD and RJS. The paper was written by RJS, SP and RC. All authors (except for NdS - since deceased) have seen and approve this manuscript.

\section{Ethics approval and consent to participate}

The A.chinenesis Red5 genotype used in this study was developed at PFR by authors MMCN and CC. 


\section{Competing interests}

The authors declare that they have no competing interests.

\section{Publisher's Note}

Springer Nature remains neutral with regard to jurisdictional claims in published maps and institutional affiliations.

\section{Author details}

'The New Zealand Institute for Plant \& Food Research Ltd (PFR), Private Bag 92169, Auckland 1142, New Zealand. ${ }^{2}$ School of Biological Sciences, University of Auckland, Private Bag 92019, Auckland 1142, New Zealand. ${ }^{3}$ PFR, Private Bag 11600, Palmerston North 4442, New Zealand. ${ }^{4}$ PFR, 412 No 1 Road, Te Puke, Bay of Plenty 3182, New Zealand. ${ }^{5}$ IGA Technology Services, Parco Scientifico e Tecnologico, Udine, Italy. 'South China Botanic Gardens, Chinese Academy of Sciences, Guangzhou 510650, Guangdong, China. ${ }^{7}$ Key Laboratory of Plant Germplasm Enhancement and Specialty Agriculture, Botanical Garden, Chinese Academy of Sciences, Wuhan 430074, Wuhan, China. ${ }^{8}$ PFR, Private Bag 4704, Christchurch 8140, New Zealand. ${ }^{9}$ Department of Agricultural and Environmental Sciences, University of Udine, Via delle Scienze 208, 33100 Udine, Italy. ${ }^{10}$ Institute for Future Environments, Queensland University of Technology (QUT), Brisbane 4001, Australia.

\section{Received: 27 August 2017 Accepted: 10 April 2018}

\section{Published online: 16 April 2018}

\section{References}

1. Arabidopsis Genome Initiative. Analysis of the genome sequence of the flowering plant Arabidopsis thaliana. Nature. 2000;408:796-815.

2. Jaillon O, Aury JM, Noel B, Policriti A, Clepet C, Casagrande A, Choisne N, Aubourg S, Vitulo N, Jubin C, et al. The grapevine genome sequence suggests ancestral hexaploidization in major angiosperm phyla. Nature. 2007:449:463-7.

3. Ming R, Hou S, Feng Y, Yu Q, Dionne-Laporte A, Saw JH, Senin P, Wang W Ly BV, KLT L, et al. The draft genome of the transgenic tropical fruit tree papaya (Carica papaya Linnaeus). Nature. 2008;452:991-6.

4. Velasco R, Zharkikh A, Affourtit J, Dhingra A, Cestaro A, Kalyanaraman A, Fontana P, Bhatnagar SK, Troggio M, Pruss D, et al. The genome of the domesticated apple (Malus x domestica Borkh.). Nat Genet. 2010;42:833-9.

5. Shulaev V, Sargent DJ, Crowhurst RN, Mockler TC, Folkerts O, Delcher AL, Jaiswal P, Mockaitis K, Liston A, Mane SP, et al. The genome of woodland strawberry (Fragaria vesca). Nat Genet. 2011;43:109-16.

6. Tomato Genome Consortium. The tomato genome sequence provides insights into fleshy fruit evolution. Nature. 2012;485:635-41.

7. D'Hont A, Denoeud F, Aury J-M, Baurens F-C, Carreel F, Garsmeur O, Noel B, Bocs S, Droc G, Rouard M, et al. The banana (Musa acuminata) genome and the evolution of monocotyledonous plants. Nature. 2012:488:213-7.

8. Xu Q, Chen L-L, Ruan X, Chen D, Zhu A, Chen C, Bertrand D, Jiao W-B, Hao $\mathrm{B}-\mathrm{H}$, Lyon MP, et al. The draft genome of sweet orange (Citrus sinensis). Nat Genet. 2013;45:59-66.

9. Chagné $D$, Crowhurst RN, Pindo $M$, Thrimawithana A, Deng $C$, Ireland $H$, Fiers $\mathrm{M}$, Dzierzon $\mathrm{H}$, Cestaro A, Fontana $\mathrm{P}$, et al. The draft genome sequence of European pear (Pyrus communis L. 'Bartlett'). PLoS One. 2014;9:e92644.

10. Deschamps S, Llaca V. Strategies for Sequence Assembly of Plant Genomes. In: Plant Genomics, Abdurakhmonov I.Y. editor. InTech, 2016.

11. Schatz MC, Witkowski J, McCombie WR. Current challenges in de novo plant genome sequencing and assembly. Genome Biol. 2012;13:243.

12. Verde I, Jenkins J, Dondini L, Micali S, Pagliarani G, Vendramin E, Paris R, Aramini V, Gazza L, Rossini L, et al. The peach v2.0 release: high-resolution linkage mapping and deep resequencing improve chromosome-scale assembly and contiguity. BMC Genomics. 2017;18:225.

13. Cai C, Wang X, Liu B, Wu J, Liang J, Cui Y, Cheng F, Wang X. Brassica rapa genome 2.0: a reference upgrade through sequence re-assembly and gene re-annotation. Mol Plant. 2016;10:649-51.

14. Huang S, Ding J, Deng D, Tang W, Sun H, Liu D, Zhang L, Niu X, Zhang X, Meng $M$, et al. Draft genome of the kiwifruit Actinidia chinensis. Nat Commun. 2013:4:2640

15. Scaglione D, Fornasiero A, Pinto C, Cattonaro F, Spadotto A, Infante R, Meneses C, Messina R, Lain O, Cipriani G, Testolin R. A RAD-based linkage map of kiwifruit (Actinidia chinensis PI.) as a tool to improve the genome assembly and to scan the genomic region of the gender determinant for the marker-assisted breeding. Tree Genet Genomes. 2015;11:1-10.
16. Zhang Q, Liu CY, Liu YF, VanBuren R, Yao XH, Zhong CH, Huang HW. Highdensity interspecific genetic maps of kiwifruit and the identification of sexspecific markers. DNA Res. 2015;22:367-75.

17. Schaffer RJ, Pilkington SM. Gene family prediction and annotation. In: The kiwifruit genome. Testolin R, Huang HW, Ferguson AR, editors. Springer International Publishing; 2016. p. 125-34.

18. Yue J, Liu J, Ban R, Tang W, Deng L, Fei Z, Liu Y. Kiwifruit information resource (KIR): a comparative platform for kiwifruit genomics. Database. 2015;2015:bav113.

19. Cheng C-Y, Krishnakumar V, Chan AP, Thibaud-Nissen F, Schobel S, Town CD. Araport11: a complete reannotation of the Arabidopsis thaliana reference genome. Plant J. 2017;89:789-804.

20. Darwish O, Shahan R, Liu Z, Slovin JP, Alkharouf NW. Re-annotation of the woodland strawberry (Fragaria vesca) genome. BMC Genomics. 2015;16:29.

21. Lee E, Helt GA, Reese JT, Munoz-Torres MC, Childers CP, Buels RM, Stein L,

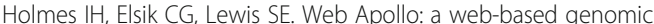
annotation editing platform. Genome Biol. 2013;14:R93.

22. Yook K, Harris TW, Bieri T, Cabunoc A, Chan J, Chen WJ, Davis P, de la Cruz N, Duong A, Fang R, et al. WormBase 2012: more genomes, more data, new website. Nucleic Acids Res. 2012:40:D735-41.

23. Cherry JM, Hong EL, Amundsen C, Balakrishnan R, Binkley G, Chan ET, Christie KR, Costanzo MC, Dwight SS, Engel SR, et al. Saccharomyces genome database: the genomics resource of budding yeast. Nucleic Acids Res. 2012:40:D700-5.

24. Lamesch P, Berardini TZ, Li D, Swarbreck D, Wilks C, Sasidharan R, Muller R, Dreher K, Alexander DL, Garcia-Hernandez M, et al. The Arabidopsis information resource (TAIR): improved gene annotation and new tools. Nucleic Acids Res. 2012;40:D1202-10.

25. Crowhurst RN, Gleave AP, MacRae EA, Ampomah-Dwamena C, Atkinson RG, Beuning LL, Bulley SM, Chagne D, Marsh KB, Matich AJ, et al. Analysis of expressed sequence tags from Actinidia: applications of a cross species EST database for gene discovery in the areas of flavor, health, color and ripening. BMC Genomics. 2008:9:351.

26. Ruan J, Jiang L, Chong Z, Gong Q, Li H, Li C, Tao Y, Zheng C, Zhai W, Turissini D, et al. Pseudo-sanger sequencing: massively parallel production of long and near error-free reads using NGS technology. BMC Genomics. 2013;14:711.

27. Boetzer $M$, Henkel $C V$, Jansen HJ, Butler D, Pirovano W. Scaffolding preassembled contigs using SSPACE. Bioinformatics. 2011;27:578-9.

28. Fraser LG, Tsang GK, Datson PM, De Silva HN, Harvey CF, Gill GP, Crowhurst RN, McNeilage MAA. Gene-rich linkage map in the dioecious species Actinidia chinensis (kiwifruit) reveals putative X/Y sex-determining chromosomes. BMC Genomics. 2009;10:102

29. Hopping ME. Flow cytometric analysis of Actinidia species. N Z J Bot. 1994; 32.85-93.

30. Simão FA, Waterhouse RM, loannidis P, Kriventseva EV, Zdobnov EM. BUSCO: assessing genome assembly and annotation completeness with single-copy orthologs. Bioinformatics. 2015;31:3210-2.

31. Wu TD, Watanabe CK. GMAP: a genomic mapping and alignment program for mRNA and EST sequences. Bioinformatics. 2005;21:1859-75.

32. Liao Y, Smyth GK, Shi W. The subread aligner: fast, accurate and scalable read mapping by seed-and-vote. Nucleic Acids Res. 2013:41:e108.

33. Quinlan AR, Hall IM. BEDTools: a flexible suite of utilities for comparing genomic features. Bioinformatics. 2010;26:841-2.

34. McAtee PA, Richardson AC, Nieuwenhuizen NJ, Gunaseelan K, Hoong L, Chen XY, Atkinson RG, Burdon JN, David KM, Schaffer RJ. The hybrid nonethylene and ethylene ripening response in kiwifruit (Actinidia chinensis) is associated with differential regulation of MADS-box transcription factors. BMC Plant Biol. 2015;15:304.

35. Friebe $B$, Zhang P, Linc G, Gill BS. Robertsonian translocations in wheat arise by centric misdivision of univalents at anaphase I and rejoining of broken centromeres during interkinesis of meiosis II. Cytogenet Genome Res. 2005; 109:293-7.

36. Mizuno H, Kawahara Y, Wu J, Katayose Y, Kanamori H, Ikawa H, Itoh T, Sasaki T, Matsumoto T. Asymmetric distribution of gene expression in the centromeric region of rice chromosome 5. Front Plant Sci. 2011:2:16.

37. Laing WA, Martinez-Sanchez M, Wright MA, Bulley SM, Brewster D, Dare AP, Rassam M, Wang D, Storey R, Macknight RC, Hellens RP. An upstream open reading frame is essential for feedback regulation of ascorbate biosynthesis in Arabidopsis. Plant Cell. 2015:27:772-86.

38. Chung BYW, Simons C, Firth AE, Brown CM, Hellens RP. Effect of 5 UTR introns on gene expression in Arabidopsis thaliana. BMC Genomics. $2006 ; 7: 120$. 
39. Celton JM, Gaillard S, Bruneau M, Pelletier S, Aubourg S, Martin-Magniette ML, Navarro L, Laurens F, Renou JP. Widespread anti-sense transcription in apple is correlated with siRNA production and indicates a large potential for transcriptional and/or post-transcriptional control. New Phytol. 2014:203:287-99.

40. Adams KL, Polyploidy WJF. genome evolution in plants. Curr Opin Plant Biol. 2005;8:135-41.

41. International Wheat Genome Sequencing Consortium. A chromosomebased draft sequence of the hexaploid bread wheat (Triticum aestivum) genome. Science. 2014;345:1251788.

42. Liu SY, Liu YM, Yang XH, Tong CB, Edwards D, Parkin IAP, Zhao MX, Ma JX, Yu JY, Huang SM, et al. The Brassica oleracea genome reveals the asymmetrical evolution of polyploid genomes. Nat Commun. 2014;5:3930.

43. Hirakawa H, Shirasawa K, Kosugi S, Tashiro K, Nakayama S, Yamada M, Kohara M, Watanabe A, Kishida Y, Fujishiro T, et al. Dissection of the octoploid strawberry genome by deep sequencing of the genomes of Fragaria species. DNA Res. 2014;21:169-81.

44. Duarte JM, Wall PK, Edger PP, Landherr LL, Ma H, Pires JC, Leebens-Mack J, dePamphilis CW. Identification of shared single copy nuclear genes in Arabidopsis, Populus, Vitis and Oryza and their phylogenetic utility across various taxonomic levels. BMC Evol Biol. 2010;10:61.

45. Naim F, Nakasugi K, Crowhurst RN, Hilario E, Zwart AB, Hellens RP, Taylor JM, Waterhouse PM, Wood CC. Advanced engineering of lipid metabolism in Nicotiana benthamiana using a draft genome and the V2 viral silencingsuppressor protein. PLoS One. 2012;7:e52717.

46. Chang S, Puryear J, Cairney JA. Simple and efficient method for isolating RNA from pine trees. Plant Mol Biol Rep. 1993;11:113-6.

47. Green MR, Sambrook J. Molecular cloning: a labarotory manual. Cold Springer Harbor, New York: Cold Springer Harbor Labarotory; 2012.

48. Gnerre S, Maccallum I, Przybylski D, Ribeiro FJ, Burton JN, Walker BJ, Sharpe T, Hall G, Shea TP, Sykes S, et al. High-quality draft assemblies of mammalian genomes from massively parallel sequence data. Proc Natl Acad Sci U S A. 2011;108:1513-8.

49. Simpson JT, Durbin R. Efficient de novo assembly of large genomes using compressed data structures. Genome Res. 2012;22:549-56.

50. Marçais G, Kingsford CA. Fast, lock-free approach for efficient parallel counting of occurrences of k-mers. Bioinformatics. 2011;27:764-70.

51. Kent WJ. BLAT - the BLAST-like alignment tool. Genome Res. 2002:12:656-64

52. Altschul SF, Gish W, Miller W, Myers EW, Lipman DJ. Basic local alignment search tool. J Mol Biol. 1990;215:403-10.

53. Langmead B, Salzberg SL. Fast gapped-read alignment with bowtie 2. Nat Methods. 2012;9:357-9.

54. Kearse M, Moir R, Wilson A, Stones-Havas S, Cheung M, Sturrock S, Buxton S, Cooper A, Markowitz S, Duran C, et al. Geneious basic: an integrated and extendable desktop software platform for the organization and analysis of sequence data. Bioinformatics. 2012;28:1647-9.

55. Dobin A, Davis CA, Schlesinger F, Drenkow J, Zaleski C, Jha S, Batut P, Chaisson M, Gingeras TRSTAR. ultrafast universal RNA-seq aligner. Bioinformatics. 2013:29:15-21.

56. Stanke M, Waack S. Gene prediction with a hidden Markov model and a new intron submodel. Bioinformatics. 2003;19(Suppl 2):ii215-25.

57. Li W, Godzik A. Cd-hit: a fast program for clustering and comparing large sets of protein or nucleotide sequences. Bioinformatics. 2006:22:1658-9.

58. Guindon S, Gascuel O. A simple, fast, and accurate algorithm to estimate large phylogenies by maximum likelihood. Syst Biol. 2003;52:696-704.

\section{Ready to submit your research? Choose BMC and benefit from:}

- fast, convenient online submission

- thorough peer review by experienced researchers in your field

- rapid publication on acceptance

- support for research data, including large and complex data types

- gold Open Access which fosters wider collaboration and increased citations

- maximum visibility for your research: over $100 \mathrm{M}$ website views per year

At BMC, research is always in progress.

Learn more biomedcentral.com/submissions 